\title{
A catalogue of Galactic supernova remnants from the Canadian Galactic plane survey
}

\section{Flux densities, spectra, and polarization characteristics ${ }^{\star}$}

\author{
R. Kothes ${ }^{1,2}$, K. Fedotov ${ }^{1,3}$, T. J. Foster ${ }^{1}$, and B. Uyanıker ${ }^{4,1}$ \\ 1 National Research Council of Canada, Herzberg Institute of Astrophysics, Dominion Radio Astrophysical Observatory, \\ PO Box 248, Penticton, British Columbia, V2A 6J9, Canada \\ e-mail: roland.kothes@nrc-cnrc.gc.ca \\ 2 Department of Physics and Astronomy, University of Calgary, 2500 University Drive N.W., Calgary, AB, Canada \\ 3 Department of Physics and Astronomy, University of Victoria, PO Box 3055, STN CSC, Victoria, BC, V8W 1A1, Canada \\ 4 Max-Planck-Institut für Radioastronomie, Auf dem Hügel 69, 53121 Bonn, Germany \\ Received 21 February 2006 / Accepted 23 May 2006
}

\section{ABSTRACT}

\begin{abstract}
We present a new catalogue containing all known Galactic supernova remnants observed in phase I and II of the Canadian Galactic Plane Survey $\left(65^{\circ} \leq \ell \leq 175^{\circ},-5^{\circ} \leq b \leq+7^{\circ}\right)$. Stokes $I$ images at $1420 \mathrm{MHz}$ of $34 \mathrm{SNRs}$ and at $408 \mathrm{MHz}$ of $36 \mathrm{SNRs}$ are displayed. From these $1^{\prime}$ and $3^{\prime}$-resolution data (respectively) we determine accurate centre positions and angular dimensions. The flux densities at 1420 and $408 \mathrm{MHz}$ are integrated and combined with values from the literature to define more accurate radio spectra. The CGPS polarization data at $1420 \mathrm{MHz}$ were examined and significant linear polarization from $18 \mathrm{SNRs}$ is found, with a modest signal from 6 others.
\end{abstract}

Key words. atlases - catalogs - polarization - ISM: supernova remnants

\section{Introduction}

As the most significant source of chemical enrichment, energy input, and cosmic ray production in the interstellar medium (ISM), supernovae play an important role in the evolution of our Galaxy. According to a simple calculation by Padmanabhan (2001) about $55 \%$ of the material in the Milky Way would have been processed by supernova explosions and their remnants. Detailed understanding of the properties of Galactic supernova remnants (SNRs) - sizes, energies, statistics, etc. - is thus of great importance in understanding the physical structure of the interstellar medium (ISM). Ultimately, such understanding rests on estimation of accurate unbiased characteristics of SNRs. However, characteristics of identified SNRs are often poorly known (for example, spectral and polarization properties and even total angular extent), and in particular distances to these objects are quite ill-defined. A problem significant at low radio frequencies and low resolution is the contribution of galactic/extragalactic point sources to the total flux, which results in overestimated flux values. Additionally the total dimension of a given SNR may not be easily found due to diffuse extended emission that cannot be detected by telescopes with low sensitivity or interferometers due to missing single antenna data. This would lead to an underestimate of the real fluxes. These effects contribute to conflicting flux density measurements for the same source at similar frequencies, which are indeed very often found throughout the literature.

Unbiased, high resolution, wide-field images of all major constituents of the Galactic ISM in the Outer Galaxy are

* Figures 2-6 and 8-10 are only available in electronic form at http://www.edpsciences.org now becoming available in the Canadian Galactic Plane Survey (CGPS, Taylor et al. 2003). The CGPS data probe the ionized, neutral, particulate, and magneto-ionic media over unprecedented spatial dynamic range, and are allowing new insights into the SNR population in our Galaxy. The synthesis telescope at the Dominion Radio Astrophysical Observatory (DRAO, Landecker et al. 2000) has been used to undertake simultaneous observations at two radio frequencies providing data for Stokes $I$ at $408 \mathrm{MHz}$ and full linear polarization information at $1420 \mathrm{MHz}$. This spectacular dataset has already been used to discover five low surface brightness SNRs (Kothes et al. 2001; Kothes 2003; Kothes et al. 2005).

In this, the first part of the CGPS SNR catalogue, we present images, newly determined flux densities at $408 \mathrm{MHz}$ and $1420 \mathrm{MHz}$ in Stokes $I$, and fractional linear polarization at $1420 \mathrm{MHz}$, for a total of 36 supernova remnants in the first and second quadrants of the Milky Way. We also include all flux densities we could find published in the literature to determine more accurate radio spectra for these SNRs.

In the second part of the catalogue (in prep.) distances, dimensions, and the spatial distribution of the SNR population will be discussed. A planned third part will contain a statistical analysis of the newly determined SNR properties.

\section{Observations and data processing}

\subsection{Observations}

The radio continuum data were obtained using the synthesis telescope (ST) of the Dominion Radio Astrophysical Observatory (DRAO, Landecker et al. 2000) as part of the Canadian Galactic 
Table 1. Newly determined centre positions and angular dimensions $\Theta_{\text {maj }}$ and $\Theta_{\min }$ of all 36 Galactic supernova remnants that can be found in the area observed for the CGPS. Equatorial coordinates (J2000) are given in [hh mm ss] for Right Ascension and in [+dd mm] for Declination. The dimensions were determined from the radio emission as seen at $1420 \mathrm{MHz}$ except for DA 530 and G156.2+5.7 (marked with*), which are only covered by the $408 \mathrm{MHz}$ survey. The designations of 1420 and $408 \mathrm{MHz}$ CGPS mosaics are given in the last column (see text).

\begin{tabular}{|c|c|c|c|c|c|c|c|}
\hline Source & $\overline{\ell \ell\left[{ }^{\circ}\right]}$ & $\overline{\overline{b\left[{ }^{\circ}\right]}}$ & $\overline{\mathrm{RA}_{J 2000}}$ & $\operatorname{Dec}_{J 2000}$ & $\overline{\Theta_{\text {maj }}\left[{ }^{\circ}\right]}$ & $\overline{\Theta_{\min }\left[{ }^{\circ}\right]}$ & Mosaic Codes \\
\hline G65.1+0.6 & 65.14 & +0.41 & 195357 & +2832 & 1.17 & 1.13 & MR1 + C7 \\
\hline G65.7+1.2 & 65.72 & +1.20 & 195213 & +2926 & 0.38 & 0.35 & $\mathrm{MR} 1+\mathrm{C} 7$ \\
\hline G67.7+1.8 & 67.74 & +1.80 & 195438 & +3128 & 0.25 & 0.20 & $\mathrm{MQ} 2+\mathrm{C} 7$ \\
\hline G68.6-1.2 & 68.59 & -1.21 & 200840 & +3036 & 0.38 & 0.38 & $\mathrm{MQ} 1+\mathrm{C} 7$ \\
\hline G69.0+2.7 & 68.81 & +2.57 & 195407 & +3247 & 1.88 & 1.48 & $\mathrm{MQ} 2+\mathrm{C} 7$ \\
\hline G69.7+1.0 & 69.69 & +1.01 & 200238 & +3243 & 0.27 & 0.23 & $\mathrm{MQ} 2+\mathrm{C} 7$ \\
\hline G73.9+0.9 & 73.83 & +0.90 & 201357 & +3608 & 0.47 & 0.43 & $\mathrm{MP} 2+\mathrm{C} 6$ \\
\hline $\mathrm{G} 74.9+1.2$ & 74.96 & +1.15 & 201603 & +3713 & 0.17 & 0.13 & $\mathrm{MP} 2+\mathrm{C} 6$ \\
\hline $\mathrm{G} 76.9+1.0$ & 76.89 & +0.97 & 202220 & +3843 & 0.15 & 0.13 & $\mathrm{MO} 2+\mathrm{C} 6$ \\
\hline $\mathrm{G} 78.2+2.1$ & 78.18 & +2.12 & 202114 & +4026 & 1.13 & 1.13 & $\mathrm{MO} 2+\mathrm{C} 6$ \\
\hline G82.2+5.3 & 82.17 & +5.29 & 201911 & +4530 & 2.05 & 1.47 & $\mathrm{MN} 2+\mathrm{C} 6$ \\
\hline G83.0-0.3 & 83.02 & -0.27 & 204654 & +4252 & 0.15 & 0.12 & $\mathrm{MM} 1+\mathrm{C} 6$ \\
\hline G84.2-0.8 & 84.20 & -0.81 & 205322 & +4327 & 0.40 & 0.32 & MM1 + C6 \\
\hline G84.9+0.5 & 84.99 & +0.50 & 205033 & +4454 & 0.13 & 0.13 & $\mathrm{MM} 1+\mathrm{C} 6$ \\
\hline G85.4+0.7 & 85.38 & +0.73 & 205057 & +4521 & 0.70 & 0.65 & MM1 + C6 \\
\hline G85.9-0.6 & 85.96 & -0.63 & 205858 & +4544 & 0.50 & 0.50 & MM1 + C6 \\
\hline G89.0+4.7 & 89.05 & +4.67 & 212731 & +5720 & 2.50 & 2.27 & ML2 + C5 \\
\hline $\mathrm{G} 93.3+6.9^{*}$ & 93.26 & +6.91 & 205219 & +5520 & 0.48 & 0.43 & C5 \\
\hline G93.7-0.2 & 93.75 & -0.36 & 212956 & +5044 & 2.00 & 1.73 & MK1 + C5 \\
\hline G94.0+1.0 & 94.00 & +1.03 & 212456 & +5154 & 0.63 & 0.55 & $\mathrm{MK} 1+\mathrm{C} 5$ \\
\hline G96.0+2.0 & 96.06 & +1.96 & 213033 & +5400 & 0.45 & 0.43 & $\mathrm{MIJ} 2+\mathrm{C} 5$ \\
\hline G106.3+2.7 & 106.35 & +2.79 & 222740 & +6057 & 1.07 & 0.70 & $\mathrm{MG} 2+\mathrm{C} 4$ \\
\hline G107.5-1.5 & 107.26 & -1.68 & 225051 & +5729 & 0.16 & 0.14 & $\mathrm{MF} 1+\mathrm{C} 4$ \\
\hline G109.1-1.0 & 109.15 & -1.02 & 230139 & +5853 & 0.58 & 0.52 & $\mathrm{MF} 1+\mathrm{C} 4$ \\
\hline G113.0+0.1 & 112.98 & +0.13 & 232654 & +6121 & 0.76 & 0.60 & $\mathrm{ME} 1+\mathrm{C} 3$ \\
\hline G114.3+0.3 & 114.32 & +0.34 & 233708 & +6158 & 1.48 & 1.27 & $\mathrm{ME} 1+\mathrm{C} 3$ \\
\hline G116.5+1.1 & 116.43 & +1.10 & 235312 & +6314 & 1.32 & 1.22 & $\mathrm{MD} 2+\mathrm{C} 3$ \\
\hline G116.9+0.2 & 116.94 & +0.19 & 235916 & +62 27 & 0.62 & 0.62 & $\mathrm{MD} 1+\mathrm{C} 3$ \\
\hline G120.1+1.4 & 120.09 & +1.41 & 002520 & +6408 & 0.18 & 0.18 & $\mathrm{MC} 2+\mathrm{C} 3$ \\
\hline G126.2+1.6 & 126.23 & +1.76 & 012204 & +6426 & 1.48 & 1.42 & $\mathrm{MB} 2+\mathrm{C} 2$ \\
\hline G127.1+0.5 & 127.11 & +0.56 & 012833 & +6308 & 0.98 & 0.88 & $\mathrm{MA} 1+\mathrm{C} 2$ \\
\hline G130.7+3.1 & 130.73 & +3.08 & 020543 & +6449 & 0.18 & 0.10 & $\mathrm{MA} 2+\mathrm{C} 2$ \\
\hline G132.7+1.3 & 132.65 & +1.56 & 021803 & +6247 & 2.07 & 1.78 & $\mathrm{MY} 2+\mathrm{C} 2$ \\
\hline $\mathrm{G} 156.2+5.7^{*}$ & 156.17 & +5.61 & 045837 & +5145 & 1.82 & 1.59 & $\mathrm{C} 0$ \\
\hline G160.9+2.6 & 160.38 & +2.74 & 050035 & +4641 & 2.48 & 2.32 & $\mathrm{MEY} 2+\mathrm{C} 0$ \\
\hline G166.0+4.3 & 166.14 & +4.25 & 052627 & +4254 & 1.60 & 0.83 & $\mathrm{MEX} 2+\mathrm{CM} 1$ \\
\hline
\end{tabular}

Plane Survey (Taylor et al. 2003). Single antenna data are incorporated into the Stokes $I$ synthesis maps to ensure accurate representation of all structures from the largest scales down to the resolution limit. The short spacing data and the DRAO ST observations are merged in Fourier space using a normalized tapering function in the overlap region. The low spatial frequency continuum data for the $408 \mathrm{MHz}$ observations were derived from the Haslam All-Sky survey (Haslam et al. 1982). For the $1420 \mathrm{MHz}$ Stokes I data, the 1.4 GHz Effelsberg survey (Reich et al. 1997) is used to augment the DRAO interferometer data. Where these data are not available, the missing spatial frequencies are derived from the 1.4 GHz Stockert $25 \mathrm{~m}$ Northern Sky Survey (Reich 1982; Reich \& Reich 1986).

Low spatial frequency data for our linear polarization observations at $1420 \mathrm{MHz}$ are currently not available. The low spatial frequency limit is determined by our shortest antenna spacing, about $13 \mathrm{~m}$. This corresponds to angular dimensions of about $1^{\circ} \times 1^{\circ} \operatorname{cosec}(\delta)(\delta$ : Declination of the observation). Hence, for SNRs larger than this in angular size we miss some of the diffuse polarized emission. The angular resolution of the final $I, Q$, and $U$ data products varies slightly across the maps as $0.82^{\prime} \times 0.82^{\prime} \operatorname{cosec}(\delta)$ and $2.8^{\prime} \times 2.8^{\prime} \operatorname{cosec}(\delta)$ at $1420 \mathrm{MHz}$ and $408 \mathrm{MHz}$, respectively.
The CGPS data are released in the form of $5^{\circ} \times 5^{\circ}$ mosaics (called "M" mosaics), at all frequencies. The larger primary beam at $408 \mathrm{MHz}$ provides a much larger sky coverage; hence at this frequency $15^{\circ} \times 15^{\circ}$ mosaics ("C $C$ " mosaics) are created in addition to the M-mosaics. The CGPS is available to the community through the Canadian Astronomy Data Centre $\left(\mathrm{CADC}^{1}\right)$. To make it easier to find each SNR in the CGPS data base we list the designation code of the mosaics in which each SNR can be found in Table 1. At the time of writing the low longitude mosaics $\left(\ell<75^{\circ}\right)$ are not yet available at $408 \mathrm{MHz}$. They will be released before the end of May, 2006.

\subsection{The SNR sample}

Our sample of Galactic SNRs is mainly based on Green's Catalogue of Galactic Supernova Remnants (Green 2004). We discuss all SNRs that are covered by phase I and phase II of the CGPS $\left(65^{\circ} \leq \ell \leq 175^{\circ}\right.$ and $\left.-5^{\circ} \leq b \leq+7^{\circ}\right)$. This includes 35 Galactic supernova remnants and 11 possible SNRs.

Three of the 35 SNRs are excluded from the catalogue. Cas A is excluded from CGPS observations for reasons of dynamic range, as discussed in Taylor et al. (2003). The

\footnotetext{
${ }^{1}$ http://cadc-ccda.hia-iha.nrc-cnrc.gc.ca/cgps
} 
observations containing Cas A are exceptionally difficult to calibrate due to the extreme brightness of this SNR. It is included in Green's catalogues of Galactic supernova remnants (Green 2004), but its emission properties will not be discussed further in this work. We will, however, include Cas A in the forthcoming papers based on this SNR catalogue, which will focus on distances and SNR statistics. The "Galactic SNR" OA 184 $(\mathrm{G} 166.2+2.5)$ has rather conclusively been shown by Foster et al. (2006) to be a misidentified H II region, as it lacks polarized emission and shines in recombination lines $\mathrm{H} 103-110 \alpha$, both observed at $\lambda 6 \mathrm{~cm}$. It is hereafter to be excluded from this and future catalogues of SNRs. G65.3+5.7 is a rather faint high latitude object which is covered by CGPS $408 \mathrm{MHz}$ measurements, but due to its low surface brightness and the high noise in its vicinity, we were not able to detect this source in our data.

Additionally we investigated the nature of the 11 "possible" SNRs listed in Green (2004). For the following seven, results were inconclusive. G71.6-0.5, G72.2-0.3, and G85.2-1.2 (Taylor et al. 1992) are confused with background emission to an extent that a distinct flux density measurement is difficult. G75.5+2.4 is suggested to be an SNR by Nichols-Bohlin \& Fesen (1993) and is quite visible in the $1420 \mathrm{MHz}$ data, but is missing in our $408 \mathrm{MHz}$ measurements. This could be caused by a flat, thermal radio spectrum and/or confusion with artifacts from nearby Cygnus A. At the positions of the candidates G69.4+1.2 (Yoshita et al. 1999), G117.7+0.6 (Hailey \& Craig 1995) and a possible remnant at $\ell=70^{\circ}$ and $b=+2^{\circ}$ (suggested by Mavromatakis et al. 2002) we find no significant radio continuum emission at either 408 or $1420 \mathrm{MHz}$.

Two of the 11 possible remnants are clearly thermal in origin. These are the SNR candidates G74.8+0.63 (Zhang 2003) and G104.7+2.8, published by Green \& Joncas (1994). They have flat radio spectra between 408 and $1420 \mathrm{MHz}$ and are also very bright infrared sources.

The following two "possible" SNRs listed in Green's catalogue (Green 2004) were added to our SNR catalogue. G83.0-0.3, discovered by Taylor et al. (1992) has a distinctly non-thermal radio spectrum and no infrared counterpart. G107.5-1.5, discovered by Kothes (2003) in CGPS data is highly polarized at $1420 \mathrm{MHz}$ (see Sect. 4 for more discussion on these sources).

We also made a literature search to find other SNRs in the CGPS area that might have been discovered since Green (2004) was published. We added two new SNRs discovered in the data of the CGPS (Kothes et al. 2005) to our sample. The only other SNR candidate we found in the literature was a possible remnant north-east of the SNR G116.5+1.1, suggested by Mavromatakis et al. (2005). However, this source was not detected in CGPS radio continuum data at either frequency, and is not included in the new SNR catalogue.

The final count in the CGPS SNR catalogue is 36 SNRs.

\subsection{Flux Integration}

Before integrating the flux density of each SNR, point sources are removed by fitting elliptical gaussians to their radio continuum emission. At $408 \mathrm{MHz}$, where the beam area is more than 10 times larger than at $1420 \mathrm{MHz}$, the fitting procedure fails for some sources due to confusion with emission from the supernova remnant. In these cases we use the catalogue of Vollmer et al. (2005) to estimate the point source flux density at $408 \mathrm{MHz}$. All sources for which $408 \mathrm{MHz}$ flux densities are determined this way are listed in Table 2. After the subtraction of the point source contribution, each SNR flux density is integrated by defining a polygon around the emission region. A twisted plane is fitted to this polygon's perimeter to determine the background contribution, which is then subtracted. The final flux density of each supernova remnant is tabulated in Table 3. This procedure was double checked by estimating a constant background from the area around the SNR. The errors in the listed flux densities consist mainly of the background uncertainty, with a contribution from a calibration error $(3 \%$ and $6 \%$ at $1420 \mathrm{MHz}$ and $408 \mathrm{MHz}$, respectively). The background uncertainty can be very high in areas of varying background emission like the Cygnus $\mathrm{X}$ region or for very faint supernova remnants where the fluctuations of the source are comparable to those in the background.

Since we merge the data observed with the DRAO ST together with the low spatial frequency observations in Fourier space it is usefull to estimate the percentage of the SNR flux density that is actually derived from the new DRAO ST observations. At $408 \mathrm{MHz}$ the uv-data is merged using a normalized tapering function between spatial dimensions of $1.7(\times 1.7 \operatorname{cosec}(\delta))$ and $3.4(\times 3.4 \operatorname{cosec}(\delta))$. Hence for at least 29 SNRs out of our sample the flux density is entirely based on the new DRAO ST observations. For HB 9, the largest SNR in our sample $(2.5 \times 2.3)$, the contribution to the flux from the Haslam survey data is less than $30 \%$. At $1420 \mathrm{MHz}$ we apply the normalized tapering function between $0.5(\times 0.5 \operatorname{cosec}(\delta))$ and $1.5(\times 1.5 \operatorname{cosec}(\delta))$ for the Effelsberg survey and between $0.75(\times 0.75 \operatorname{cosec}(\delta))$ and $1.5(\times 1.5 \operatorname{cosec}(\delta))$ for the Stockert survey. Hence, for at least 15 SNRs the flux density is entirely derived from the new DRAO ST observation. For HB 9 the contribution of the Effelsberg survey is about $70 \%$, for VRO $42.01 .05(1.6 \times 0.8)$ this number drops to less than $30 \%$.

\section{Results}

\subsection{The structure of the SNRs in Stokes I and their spectra}

In Figs. 1 and 2-6 we display Stokes $I$ images of all SNRs within the bounds of the CGPS at $1420 \mathrm{MHz}\left(65^{\circ}<\ell<175^{\circ},-3^{\circ} .5<\right.$ $b<+5.5)$ and $408 \mathrm{MHz}\left(65^{\circ}<\ell<175^{\circ},-6.5<b<+8^{\circ} .5\right)$. For G89.0+4.7 (HB 21) and G82.2+5.3 (W 63), two large high latitude SNRs not fully covered by the CGPS at the higher frequency, we include data from the archive of the DRAO synthesis telescope to augment the $1420 \mathrm{MHz}$ measurements. For the SNRs G93.3+6.9 (DA 530) and G156.2+5.7 (not observed in the CGPS at $1420 \mathrm{MHz}$ due to their high latitude) we only provide $408 \mathrm{MHz}$ images and flux density values. As the field of view is more than ten times larger at $408 \mathrm{MHz}$ than at $1420 \mathrm{MHz}$, these two SNRs are covered at the lower frequency. The newly determined centres of the emission regions and the total angular dimensions of all the SNRs are listed in Table 1, and flux densities at 408 and $1420 \mathrm{MHz}$ appear in Table 3. The values specified for the dimensions are not the actual major and minor axes of the emission region, but their maximum extents in $\ell$ and $b$, which may include outbreaks, etc. The centres of the SNRs were derived from these dimensions. Hence, these are not the actual positions of the supernova explosions that lead to the SNRs, but the centres of the currently observed emission. The error in dimensions and positions for most SNRs is about 1'.

In Figs. 7 and 8 to 10 we display the radio continuum spectra of 34 of our 36 SNRs. For the SNR G113.0+0.1 no reliable flux density value is available, and for SNR G68.6-1.2 only a $1420 \mathrm{MHz}$ measurement can be determined. To more accurately define the spectra we searched the literature for flux densities (which we judge to be reliable) for many frequencies. 
Table 2. $408 \mathrm{MHz}$ flux density values for point sources seen along with the SNR. These fluxes are predicted from the spectra published by Vollmer et al. (2005), except for ${ }^{*}$, where the Northern VLA Sky Survey (NVSS, Condon et al. 1998) was used at $1420 \mathrm{MHz}$, and the Westerbork Northern Sky Survey (WENSS, Rengelink et al. 1997) at 327 MHz.

\begin{tabular}{lrrlcc}
\hline \hline SNR & $\ell\left[^{\circ}\right]$ & $b\left[^{\circ}\right]$ & RA $_{J 2000}$ & Dec $_{J 2000}$ & $S_{408}[\mathrm{mJy}]$ \\
\hline G65.7+1.2 & 65.61 & +1.08 & 195225 & +2917 & $73 \pm 7$ \\
& 65.69 & +1.26 & 195154 & +2926 & $500 \pm 60$ \\
& 65.92 & +1.19 & 195243 & +2936 & $260 \pm 50$ \\
G67.7+1.8 & $67.73^{*}$ & +1.89 & 195415 & +3131 & $156 \pm 16$ \\
G69.0+2.7 & 68.65 & +2.93 & 195215 & +3250 & $1000 \pm 50$ \\
& 68.75 & +2.03 & 195610 & +3227 & $210 \pm 20$ \\
& 68.81 & +2.12 & 195557 & +3233 & $200 \pm 40$ \\
& 69.25 & +2.83 & 195408 & +3318 & $400 \pm 20$ \\
G73.9+0.9 & 73.81 & +0.97 & 201337 & +3610 & $890 \pm 40$ \\
G82.2+5.3 & 81.26 & +5.03 & 201733 & +4436 & $260 \pm 60$ \\
& 81.77 & +5.34 & 201741 & +4512 & $260 \pm 40$ \\
& 82.25 & +5.51 & 201824 & +4541 & $270 \pm 30$ \\
& 82.44 & +4.84 & 202211 & +4528 & $680 \pm 90$ \\
G84.2-0.8 & 84.32 & -0.81 & 205347 & +4333 & $480 \pm 20$ \\
G84.9+0.5 & 84.94 & +0.41 & 205046 & +4448 & $710 \pm 140$ \\
& 85.03 & +0.48 & 205047 & +4455 & $170 \pm 20$ \\
G85.4+0.7 & 85.61 & +0.64 & 205211 & +4528 & $280 \pm 80$ \\
G85.9-0.6 & 85.80 & -0.66 & 205830 & +4446 & $3000 \pm 500$ \\
& 86.10 & -0.50 & 205856 & +4506 & $26 \pm 11$ \\
G89.0+4.7 & 88.55 & +4.23 & 204639 & +5000 & $340 \pm 70$ \\
& 89.02 & +5.66 & 204122 & +5115 & $370 \pm 40$ \\
& 89.34 & +5.41 & 204353 & +5121 & $310 \pm 30$ \\
G94.0+1.0 & 94.12 & +1.21 & 212441 & +5207 & $2800 \pm 560$ \\
G96.0+2.0 & 95.89 & +2.03 & 212923 & +5356 & $190 \pm 20$ \\
G116.9+0.2 & 116.94 & -0.01 & 235936 & +6215 & $350 \pm 40$ \\
G130.7+3.1 & 130.79 & +3.18 & 020632 & +6454 & $520 \pm 40$ \\
G160.9+2.6 & 159.88 & +2.00 & 045524 & +4637 & $340 \pm 40$ \\
& 160.11 & +2.42 & 045809 & +4642 & $122 \pm 3$ \\
& 161.14 & +2.77 & 050325 & +4606 & $226 \pm 8$ \\
& 161.35 & +2.90 & 050444 & +4601 & $180 \pm 20$ \\
\hline
\end{tabular}

It is to be noted that we did not add any other flux densities determined with CGPS data by other authors to be consistent with the method of flux determination. An error weighted linear function is fitted to each $\log S-\log v$ plot, and the final spectral index $\alpha_{\text {all }}$ is thus calculated. We also determine $\alpha_{\mathrm{CGPS}}$, calculated between the two CGPS flux densities only (listed in Table 3). A numberfrequency distribution of spectral index values $\alpha_{\text {all }}$ is displayed in Fig. 11.

\subsection{Polarization properties}

Out of the 36 supernova remnants in our catalogue 24 appear to be polarized at $1420 \mathrm{MHz}$ in the CGPS data. Polarized intensity images of 18 of these SNRs are displayed in Figs. 12 to 13. For the others, polarized emission is too ill-defined to display in a figure. The polarized intensity PI was calculated from the Stokes $Q$ and $U$ emission via: $\mathrm{PI}=\left(Q^{2}+U^{2}-(1.2 \sigma)^{2}\right)^{0.5}$. The third term is a first order correction for noise bias (Wardle \& Kronberg 1974) and $\sigma \approx 0.03 \mathrm{~K}$ is the rms noise in the Stokes $Q$ and $U$ images. Integrated polarized intensities, percentage polarization, and peak polarization for all SNRs that show significant related polarized emission are listed in Table 4. All supernova remnants should be intrinsically polarized up to $70 \%$. However, at $1420 \mathrm{MHz}$ polarized emission suffers significantly from beam depolarization (caused by Faraday rotation). A rotation measure RM of only $\pm 36 \mathrm{rad} / \mathrm{m}^{2}$ is required to rotate the polarization angle by $\pm \frac{\pi}{2}$, and two polarization angles perpendicular to each other within one beam would cancel. It is quite remarkable that we still detect linearly polarized emission from so many supernova remnants even as far away as G67.7+1.8 (see individual notes).

\section{Notes on individual objects}

Supernova remnants are divided into three groups based on their appearance and spectral properties. Shell-type supernova remnants are the actual remains of the explosion and appear as highly linearly polarized limb brightened shells of radio continuum emission. An adiabatically expanding pure shell-type remnant is expected to have a non-thermal radio spectrum with a spectral index $\alpha$ of about $\sim-0.5\left(S \sim v^{\alpha}\right)$. Younger, freely expanding shell-type remnants, like Tycho in our sample, have slightly steeper spectra, and when the remnant becomes older and radiative, the spectrum might flatten. Pulsar wind nebulae, also known as filled-centre, crab-like, or plerion-type SNRs, are produced by a rapidly spinning pulsar in their centre that releases a wind of particles and magnetic field in its surroundings. They have flat radio spectra with indices between 0.0 and -0.3 , which is similar to optically thin thermal emission like that observed from $\mathrm{H}$ II regions. The non-thermal emission coming from pulsar wind nebulae can be identified because this emission is highly linearly polarized. The third group are the so-called combined type SNRs which contain both, a shell-type remnant and a pulsar wind nebula.

There are 36 supernova remnants listed in our catalogue. Out of these 28 objects are considered to be pure shell-type remnants, 4 are believed to be pure pulsar wind nebulae (PWNe), 2 objects contain both, and another 2 are unknown (see Green 2004). These numbers are reflected in the histogram displayed 
Table 3. Flux densities integrated at $1420 \mathrm{MHz}$ and $408 \mathrm{MHz}$ taken from the CGPS. Spectral indices $\alpha$ are listed assuming $S \sim \nu^{\alpha}$. The spectral index $\alpha_{\text {all }}$ fitted to all flux densities by an error-weighted least square fit is listed in Col. 4 and the spectral index calculated between our two values $\alpha_{\text {CGPS }}$ is listed in Col. 5. The flux densities we used to calculate $\alpha_{\text {all }}$ were taken from the references listed in Col. 6 and references therein.

\begin{tabular}{|c|c|c|c|c|c|}
\hline Source & $\overline{S_{1420}[\mathrm{Jy}]}$ & $\overline{S_{408}[\mathrm{Jy}]}$ & $\overline{\overline{\alpha_{\text {all }}}}$ & $\alpha_{\mathrm{CGPS}}$ & References \\
\hline G65.1+0.6 & $3.9 \pm 0.5$ & $9.1 \pm 1.0$ & $-0.61 \pm 0.09$ & $-0.69 \pm 0.14$ & 1 \\
\hline G65.7+1.2 & $4.0 \pm 0.2$ & $6.5 \pm 0.6$ & $-0.49 \pm 0.04$ & $-0.38 \pm 0.08$ & $2,3,4$ \\
\hline G67.7+1.8 & $0.68 \pm 0.04$ & $1.1 \pm 0.1$ & $-0.49 \pm 0.05$ & $-0.39 \pm 0.09$ & 5 \\
\hline G68.6-1.2 & $0.57 \pm 0.08$ & - & - & - & - \\
\hline G69.0+2.7 & $56 \pm 5$ & $72 \pm 7$ & $-0.45 \pm 0.03$ & $-0.20 \pm 0.10$ & 6 \\
\hline G69.7+1.0 & $1.5 \pm 0.1$ & $3.2 \pm 0.4$ & $-0.70 \pm 0.06$ & $-0.61 \pm 0.12$ & 7 \\
\hline G73.9+0.9 & $7.6 \pm 0.6$ & $10.0 \pm 1.7$ & $-0.23 \pm 0.03$ & $-0.22 \pm 0.15$ & 8,9 \\
\hline G74.9+1.2 & $7.1 \pm 1.1$ & $11.9 \pm 0.9$ & $-0.29 \pm 0.02$ & $-0.42 \pm 0.14$ & $3,9,10,11$ \\
\hline G76.9+1.0 & $1.35 \pm 0.07$ & $2.3 \pm 0.2$ & $-0.60 \pm 0.02$ & $-0.44 \pm 0.08$ & 12 \\
\hline $\mathrm{G} 78.2+2.1$ & $226 \pm 19$ & $500 \pm 35$ & $-0.51 \pm 0.03$ & $-0.64 \pm 0.09$ & $9,11,13$ \\
\hline $\mathrm{G} 82.2+5.3$ & $93 \pm 5$ & $144 \pm 12$ & $-0.48 \pm 0.04$ & $-0.36 \pm 0.08$ & 14,15 \\
\hline G83.0-0.3 & $0.8 \pm 0.1$ & $1.2 \pm 0.3$ & $-0.44 \pm 0.11$ & $-0.32 \pm 0.23$ & 5 \\
\hline G84.2-0.8 & $7.2 \pm 0.8$ & $14.5 \pm 0.5$ & $-0.50 \pm 0.04$ & $-0.56 \pm 0.10$ & 16,17 \\
\hline G84.9+0.5 & $0.70 \pm 0.08$ & $0.77 \pm 0.22$ & $-0.35 \pm 0.04$ & $-0.08 \pm 0.25$ & 5 \\
\hline G85.4+0.7 & $2.3 \pm 0.2$ & $2.9 \pm 0.5$ & $-0.19 \pm 0.16$ & $-0.19 \pm 0.16$ & - \\
\hline G85.9-0.6 & $2.2 \pm 0.8$ & $3.0 \pm 1.3$ & $-0.23 \pm 0.47$ & $-0.23 \pm 0.47$ & - \\
\hline G89.0+4.7 & $183 \pm 9$ & $259 \pm 19$ & $-0.38 \pm 0.03$ & $-0.27 \pm 0.07$ & $2,15,18$ \\
\hline G93.3+6.9 & - & $10.5 \pm 0.7$ & $-0.45 \pm 0.04$ & - & 19,20 \\
\hline G93.7-0.2 & $35 \pm 4$ & $67 \pm 6$ & $-0.65 \pm 0.03$ & $-0.52 \pm 0.12$ & $21,22,23,24$ \\
\hline G94.0+1.0 & $11.3 \pm 1.0$ & $20 \pm 2$ & $-0.48 \pm 0.02$ & $-0.32 \pm 0.12$ & $2,21,22,23,25$ \\
\hline G96.0+2.0 & $0.24 \pm 0.02$ & $0.42 \pm 0.06$ & $-0.45 \pm 0.13$ & $-0.45 \pm 0.13$ & 26 \\
\hline $\mathrm{G} 106.3+2.7$ & $4.8 \pm 0.5$ & $8.6 \pm 1.0$ & $-0.61 \pm 0.07$ & $-0.47 \pm 0.12$ & 27 \\
\hline G107.5-1.5 & $0.07 \pm 0.01$ & $0.14 \pm 0.02$ & $-0.56 \pm 0.16$ & $-0.56 \pm 0.16$ & 28 \\
\hline G109.1-1.0 & $17.4 \pm 1.2$ & $26 \pm 3$ & $-0.50 \pm 0.04$ & $-0.32 \pm 0.11$ & $29,30,31,32$ \\
\hline G114.3+0.3 & $5.4 \pm 0.8$ & - & $-0.49 \pm 0.25$ & - & 33 \\
\hline G116.5+1.1 & $10.3 \pm 0.70$ & $12.5 \pm 1.6$ & $-0.53 \pm 0.08$ & $-0.16 \pm 0.11$ & 33 \\
\hline G116.9+0.2 & $7.0 \pm 0.8$ & $10.5 \pm 0.8$ & $-0.61 \pm 0.03$ & $-0.33 \pm 0.13$ & $2,21,33,34,35$ \\
\hline G120.1+1.4 & $40.5 \pm 1.5$ & $86 \pm 5$ & $-0.65 \pm 0.01$ & $-0.61 \pm 0.06$ & 36 \\
\hline G126.2+1.6 & $6.4 \pm 1.1$ & $5.7 \pm 0.7$ & $-0.48 \pm 0.06$ & $0.10 \pm 0.17$ & $15,37,38$ \\
\hline $\mathrm{G} 127.1+0.5$ & $9.7 \pm 0.6$ & $15.9 \pm 1.0$ & $-0.45 \pm 0.02$ & $-0.40 \pm 0.07$ & $37,38,39$ \\
\hline $\mathrm{G} 130.7+3.1$ & $31.9 \pm 1.0$ & $32.2 \pm 2.0$ & $-0.07 \pm 0.01$ & $-0.01 \pm 0.05$ & $40,41,42$ \\
\hline $\mathrm{G} 132.7+1.3$ & $29.4 \pm 2.7$ & $61 \pm 9$ & $-0.66 \pm 0.02$ & $-0.59 \pm 0.14$ & $15,21,43$ \\
\hline G156.2+5.7 & - & $8.1 \pm 1.3$ & $-0.53 \pm 0.17$ & - & 44 \\
\hline $\mathrm{G} 160.9+2.6$ & $54.0 \pm 2.9$ & $102 \pm 7$ & $-0.64 \pm 0.02$ & $-0.51 \pm 0.07$ & $15,45,46$ \\
\hline $\mathrm{G} 166.0+4.3$ & $5.1 \pm 0.4$ & $8.1 \pm 0.9$ & $-0.37 \pm 0.03$ & $-0.37 \pm 0.11$ & 2,47 \\
\hline
\end{tabular}

References for the flux densities are: (1) Landecker et al. (1990), (2) Willis (1973), (3) Dickel \& DeNoyer (1975), (4) Landecker \& Caswell (1983), (5) Taylor et al. (1992), (6) Mantovani et al. (1985), (7) Reich et al. (1988, Wolfgang Reich, priv. communication), (8) Reich et al. (1986), (9) Pineault \& Chastenay (1990), (10) Weiler \& Shaver (1978), (11) Wendker et al. (1991), (12) Landecker et al. (1993), (13) Higgs et al. (1977), (14) Higgs et al. (1991), (15) Reich et al. (2003), (16) Matthews et al. (1977), (17) Matthews \& Shaver (1980), (18) Hirabayashi \& Takahashi (1972), (19) Haslam et al. (1980), (20) Lalitha et al. (1984), (21) Velusamy \& Kundu (1974), (22) Mantovani et al. (1982), (23) Landecker et al. (1985), (24) Mantovani et al. (1991), (25) Goss et al. (1984), (26) Kothes et al. (2005), (27) Pineault \& Joncas (2000), (28) Kothes (2003), (29) Hughes et al. (1981), (30) Downes (1983), (31) Sofue et al. (1983), (32) Hughes et al. (1984), (33) Reich \& Braunsfurth (1981), (34) Angerhofer et al. (1977), (35) Landecker et al. (1982b), (36) Klein et al. (1979), (37) Reich et al. (1979), (38) Joncas et al. (1989), (39) Pauls (1977), (40) Green et al. (1975), (41) Green (1986), (42) Morsi \& Reich (1987), (43) Landecker et al. (1987), (44) Reich et al. (1992), (45) Dwarakanath et al. (1982), (46) Roger et al. (1999), (47) Landecker et al. (1982a).

in Fig. 11, which shows a peak at a spectral index of -0.5 indicating that most of the remnants discussed in our catalogue are adiabatically expanding shell-type SNRs. This is not to say that most SNRs in the first and second quadrant are in this intermediate phase of their evolution; rather, we believe that a Malmquisttype bias is at work. The adiabatic phase marks where the shock is most efficiently interacting with swept-up material, and where emission of synchrotron radiation is significant. This causes a predilection whereby more mature adiabatic remnants are detected in radio emission than very old ones.

\section{G65.1+0.6}

G65.1+0.6 is a very faint object of large angular extent (see Fig. 1). It consists of two shells on top of diffuse emission, with the southern shell more pronounced than the northern. Its steep radio spectrum (Fig. 7) would indicate a rather young shell-type remnant. Since it appears to be very diffuse without a sharp outer edge it is possible that the remnant is expanding inside a void in the H I, probably a stellar wind bubble, or just a very low density environment. This way it could be of high age, still showing the characteristics of a young almost freely expanding shell-type SNR. We did not detect any polarized emission from this object, which is likely the result of its low signal-to-noise ratio.

\section{G65.7+1.2 (DA 495)}

DA 495 is believed to be a pulsar wind nebula (Landecker \& Caswell 1983) even though its spectrum is unusually steep for such an object (see Figs. 1 and 7). We found this supernova remnant to be a bit larger than the first impression would predict. While the area of detectable radio continuum emission in 

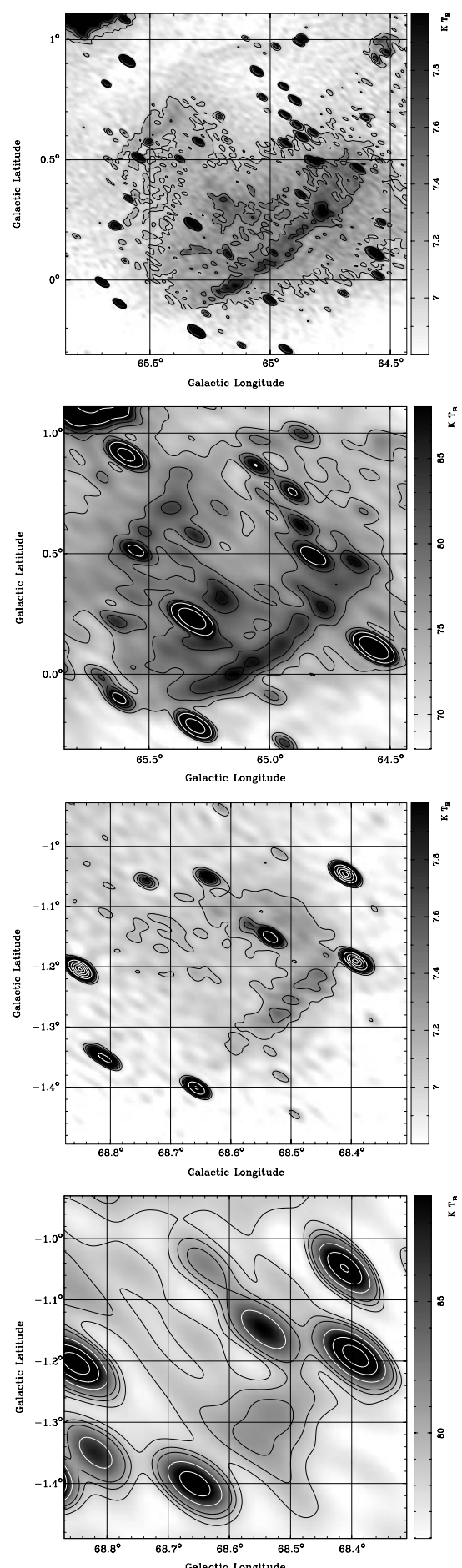
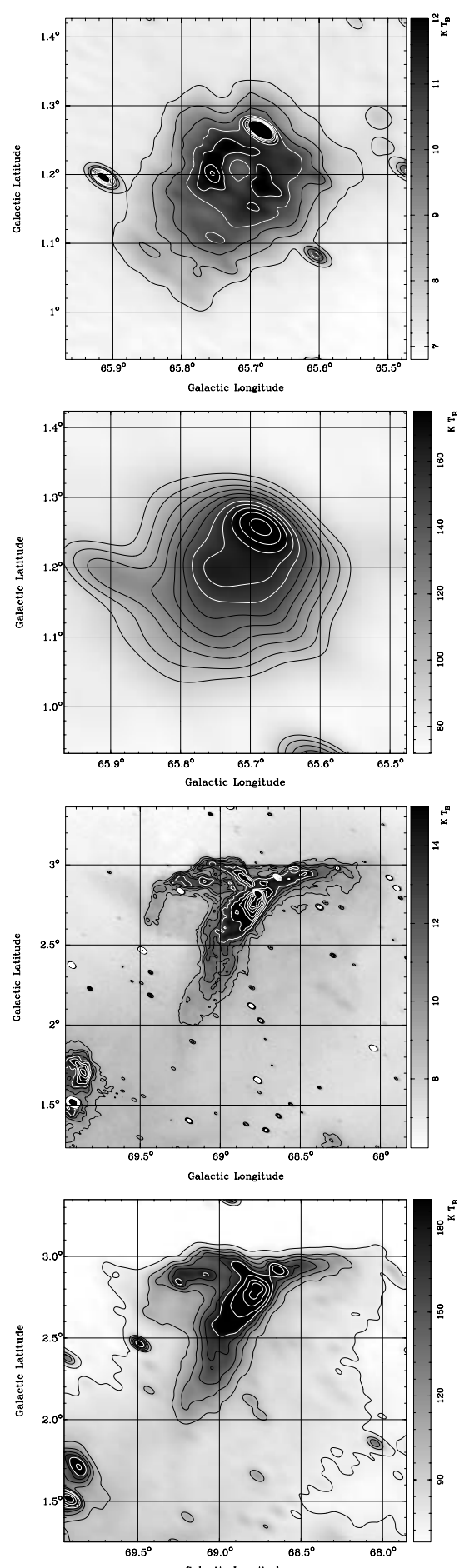
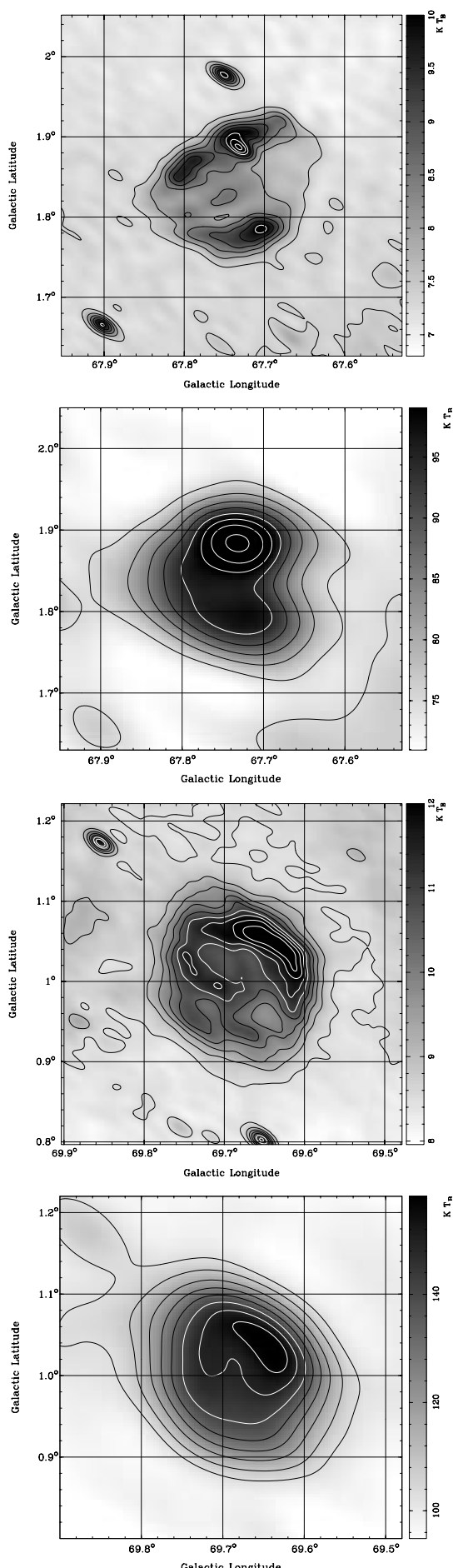

Fig. 1. Total power images at $1420 \mathrm{MHz}$ (first and third row) and $408 \mathrm{MHz}$ (second and fourth row) of the supernova remnants G65.1+0.6, G65.7+1.2 (DA 495), G67.7+1.8, G68.6-1.2, G69.0+2.7 (CTB 80), and G69.7+1.0.

shell-type supernova remnants is known to have a very sharp edge outside the shock wave, PWNe fade away slowly with larger radii. This is certainly the case for DA 495 since it seems to sit on a diffuse emission plateau which is a bit larger than its bright radio continuum emission listed in Table 1 . Since this diffuse emission is most likely included in published flux densities (especially those observed with low resolution) we included it in our measurements listed in Table 3 as well. Point source contribution to the total flux density could be significant for this object especially at the lower frequencies, so that our spectral index should be a lower limit. DA 495 is highly polarized (see Fig. 12), probably the result of a short distance and low internal depolarization.

\section{G67.7+1.8}

G67.7+1.8 has a typical double shell structure and belongs to the class of the so-called barrel-shaped SNRs (see Fig. 1). As already noted by Taylor et al. (1992) the bright point source 


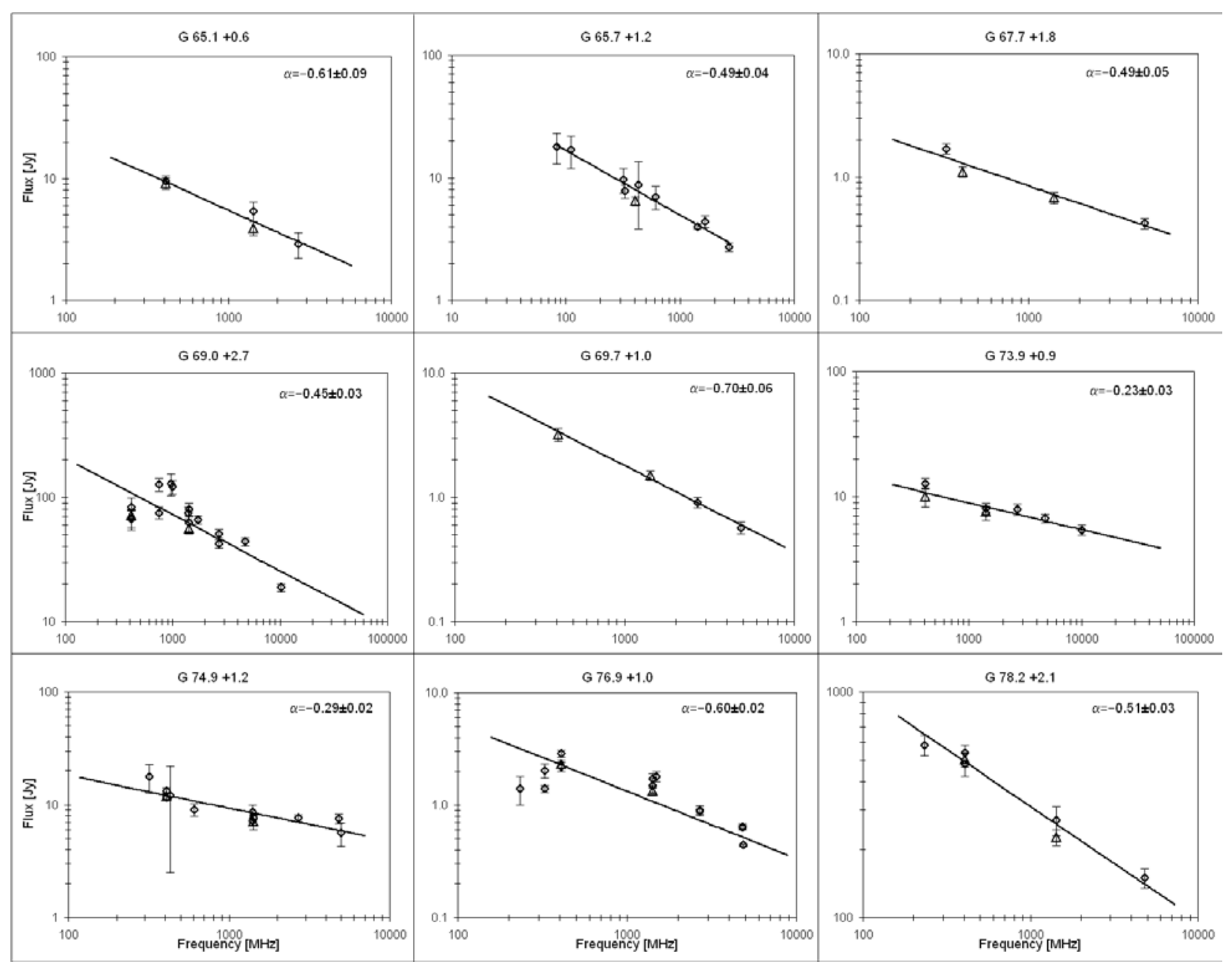

Fig. 7. The radio continuum spectra of the SNRs G65.1+0.6, G65.7+1.2 (DA 495), G67.7+1.8, G69.0+2.7 (CTB 80), G69.7+1.0, G73.9+0.9, G74.9+1.2 (CTB 87), G76.9+1.0, and G78.2+2.1 (DR 4, $\gamma$ Cygni). The CGPS flux densities are represented by triangles.

located on the northern shell of G67.7+1.8 is most likely of extragalactic origin. We used catalogued flux densities of this source to calculate its flux at $408 \mathrm{MHz}$ and subtracted it from our integrated flux value for the SNR (see Table 2). We also calculate its flux at $327 \mathrm{MHz}$ to subtract it from the SNR's integrated flux density published by Taylor et al. (1992) before calculating the spectrum in Fig. 7. Our two new flux density values confirm G67.7+1.8's nature as a shell-type supernova remnant. A spectral index of $\alpha=-0.49$ (Fig. 7) indicates a mature adiabatically expanding SNR. We detect a high degree of linear polarization from the southern shell of this object, while the northern shell seems to be unpolarized (see Fig. 12).

\section{G68.6-1.2}

G68.6-1.2 was identified as a supernova remnant by Reich et al. (1988) because of its linearly polarized radio continuum emission at $2695 \mathrm{MHz}$. The image displayed in Fig. 1 is the first one to be published of this illusive object. It is a very diffuse almost circular SNR with a smooth shell-like feature to the west. Unfortunately, we are not able to determine any spectral information, since our $1420 \mathrm{MHz}$ value is the only reliable flux density available. With this information alone we cannot tell whether this object is a pulsar wind nebula or a shell-type SNR; however, its appearance would certainly point to the latter.
At $408 \mathrm{MHz}$ the signal-to-noise ratio of this object is too low for a clear detection and we did not detect any significant polarized emission at $1420 \mathrm{MHz}$, probably the result of the low signal-tonoise ratio.

\section{G69.0+2.7 (CTB 80)}

CTB 80 is a very complex object of large angular size (see Fig. 1). It is rather difficult to determine a reliable value for its dimensions. Especially to the south it contains many diffuse filaments, which may not be included in all published flux density values especially those with low sensitivity and/or low resolution. To the south the remnant also seems to slowly fade away overlapping with diffuse thermal emission which makes it virtually impossible to determine its edge in that direction. The difficulty in determining its true extent is also reflected in the large scatter in its radio continuum spectrum (see Fig. 7). The overall spectrum is typical of an adiabatic shell-type remnant. CTB 80 is believed to be a combined type supernova remnant consisting of (a) shell-type remnant(s) and a pulsar wind nebula. CTB 80 is highly polarized at $1420 \mathrm{MHz}$ (see Fig. 12). Prominent in the north-east is linearly polarized emission that seems to extend beyond the total power emission, indicating that the actual SNR is even larger than it appears in the continuum. 


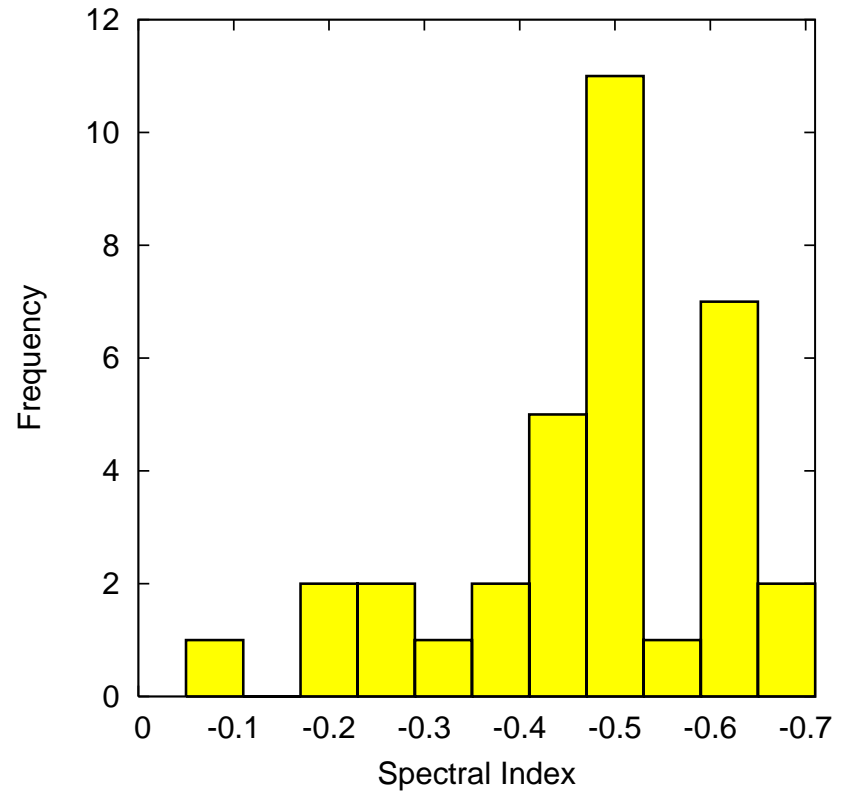

Fig. 11. Distribution of the spectral index $\alpha_{\text {all }}$ (see Table 3 ) of all supernova remnants listed in this publication.

\section{G69.7+1.0}

The supernova remnant G69.7+1.0 heretofore has had only poorly determined characteristics. Our $1420 \mathrm{MHz}$ observation is the first detailed image available for this SNR (see Fig. 1). The remnant is almost circular and of small angular size, with a bright shell to the north-west. The spectral index of $\alpha=-0.70$ (see Fig. 7) is rather steep and indicates a relatively young object. G69.7+1.0 is unpolarized in our $1420 \mathrm{MHz}$ data, indicating a large distance resulting in high beam depolarization and/or strong internal effects.

\section{G73.9+0.9}

The supernova remnant G73.9+0.9 is a rather diffuse object in our $1420 \mathrm{MHz}$ maps (see Fig. 2). It is considered to be a shelltype remnant and consists of a shell-like feature to the west with a sharp outer boundary and a centrally peaked nebula to the east of it. The spectrum is very flat with $\alpha=-0.23$ (see Fig. 7). A possible explanation is that the feature in the centre of the source is a pulsar wind nebula. In this case G73.9+0.9 would be a combined-type SNR. A comparison of our $1420 \mathrm{MHz}$ data with the $408 \mathrm{MHz}$ observations does not reveal any new information, because the resolution at $408 \mathrm{MHz}$ is not sufficient. The bright unresolved source at the northern edge of the remnant and the bright source on top of the northern tip of the shell were considered unrelated background sources and were subtracted. There seems to be faint polarization in our data at the position of this SNR (see Fig. 12), but a correlation is difficult to confirm.

\section{G74.9+1.2 (CTB 87)}

CTB 87 consists of a bright kidney shaped central part sitting on diffuse extended emission (see Fig. 2) and is considered to be a pulsar wind nebula. Our flux measurements confirm the flat radio spectrum of this source with a spectral index of $\alpha=-0.29$ (see Fig. 7). We do detect faint linear polarization from this object (see Fig. 12). The integrated percentage polarization of $1.7 \%$ and the peak polarization of $5 \%$ are rather low and likely only detectable because CTB 87 is extremely bright. The detected polarization cannot be instrumental, since there is a depolarization canal going right through the centre of it, indicating a beam depolarization effect while instrumental polarization would be very smooth, showing the same structure as the total power emission.

\section{G76.9+1.0}

G76.9+1.0 (see Fig. 2) is believed to be a pure pulsar wind nebula even though its radio spectrum (see Fig. 7) is rather steep for such an object (Landecker et al. 1993). This makes it very similar to DA 495. Like this PWN it has no sharp outer boundary, but the emission seems to fade away slowly with larger radius. In our $1420 \mathrm{MHz}$ data this source is only weakly polarized (see Fig. 12), probably the result of a large distance and/or confusion with the complex and very bright Cygnus $\mathrm{X}$ region.

\section{G78.2+2.1 (DR 4, y Cygni)}

$\gamma$ Cygni is a very bright almost circular supernova remnant with a rather complex substructure (see Fig. 2). Its overall radio spectrum with a spectral index of $\alpha=-0.51$ (see Fig. 7) and the shell-like - almost barrel-shape - appearance classifies it as a typical adiabatically expanding shell-type SNR. We do detect faint polarized emission from the SNR but most of it seems to be instrumental, since the total power emission is very bright. But there might be some faint genuine emission coming from the southern shell with a peak polarization of about $2 \%$ (see Table 4).

\section{G82.2+5.3 (W63)}

W 63 is a very large nearby high latitude supernova remnant, which is considered to be of shell-type (Fig. 2). It is difficult to determine its true extent, because it is not clear which of the filaments surrounding the main source actually belongs to the SNR. This fact is reflected in the large scatter in its overall radio spectrum (Fig. 8). The spectral index of -0.48 and its nicely limbbrightened structure indicate that it is a pure shell-type remnant. There is almost no significant linearly polarized emission detected in the CGPS (see Table 4). However, since this SNR is located at a high Galactic Latitude, with an angular extent of almost $2^{\circ}$ it is reasonable to assume that it does not suffer much from foreground beam depolarization. Thus the polarized emission might be rather smooth, which could be impossible to detect with our synthesis telescope due to missing low spatial frequency data.

\section{G83.0-0.3}

G83.0-0.3 is listed by Green (2004) as a possible SNR originally reported by Taylor et al. (1992). No study since then has confirmed its non-thermal nature. It has the structure of a shell of small angular size (Fig. 2). It lies within the confusing and highly structured background of Cygnus X, and sits atop a filament of thermal continuum emission. A single, flat-spectrum point source is within the remnant's boundary, and after removing its flux, we integrated 408, 1420, and $2695 \mathrm{MHz}(11 \mathrm{~cm}$ Effelsberg Survey, Fürst et al. 1990) data and used the point source corrected $327 \mathrm{MHz}$ value from Taylor et al. (1992) to confirm a non-thermal spectrum for G83.0-0.3 (Fig. 8). Taylor et al. (1992) measured a flux at $4850 \mathrm{MHz}$ from the Green Bank survey (Condon et al. 1989). However, in these data we find that 

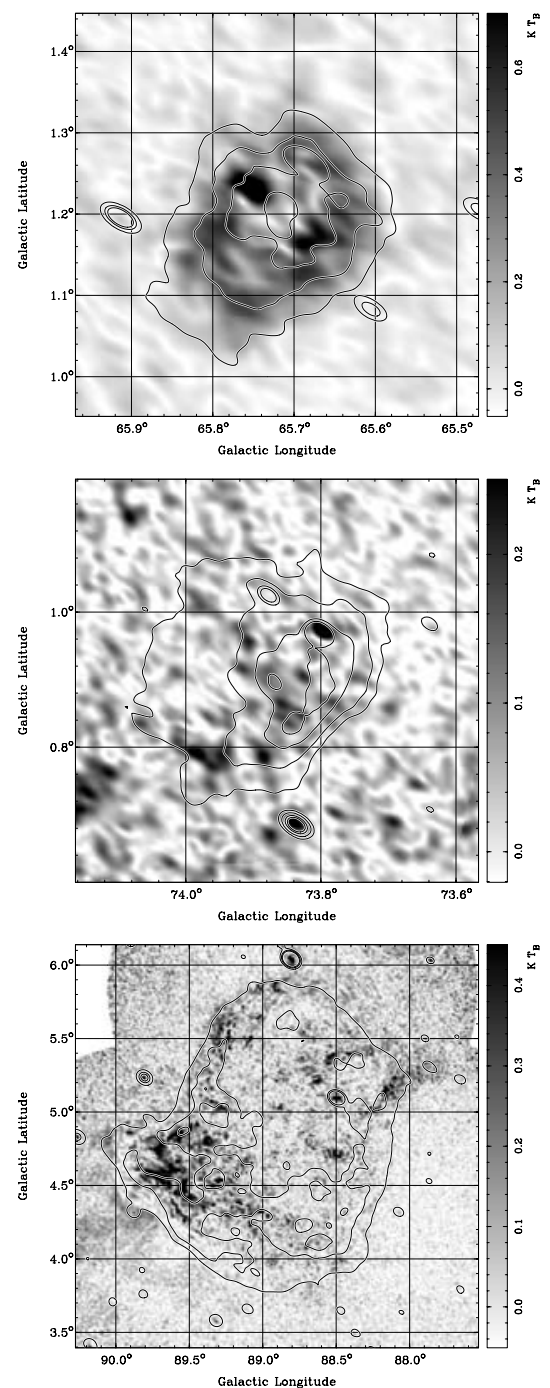
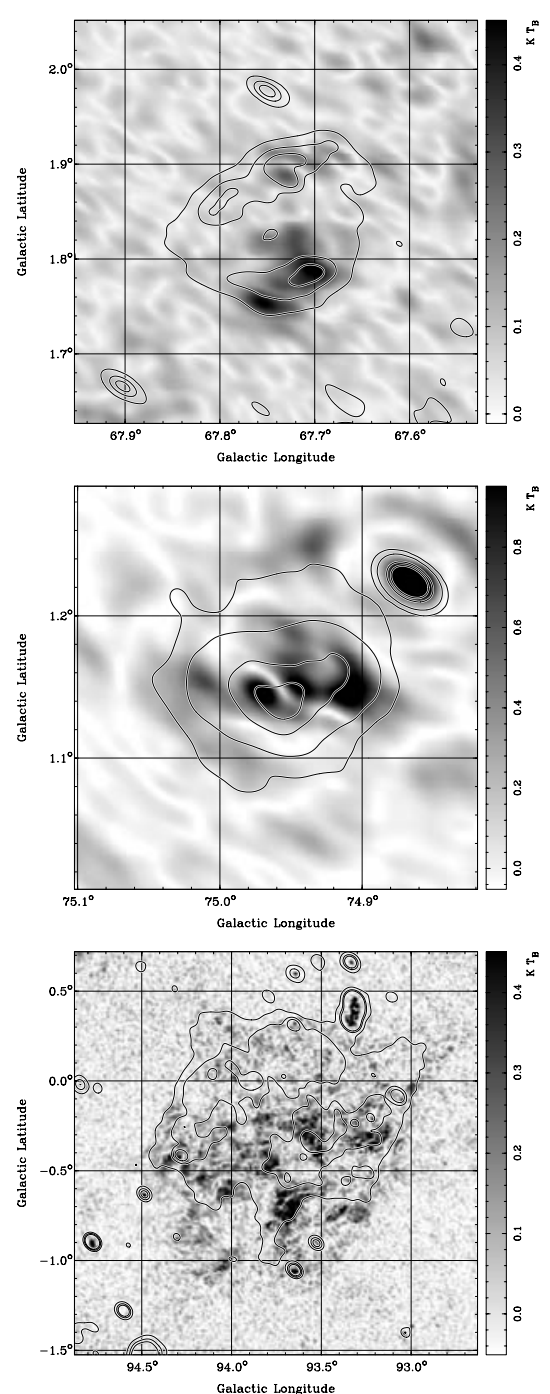
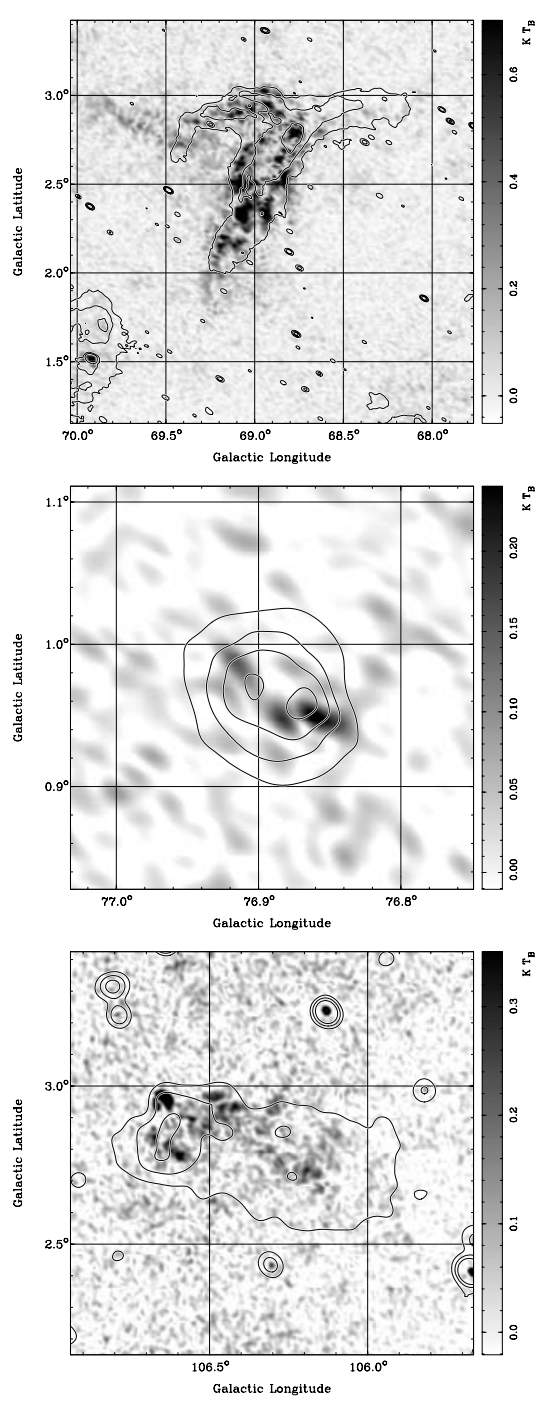

Fig. 12. Images of polarized intensity at $1420 \mathrm{MHz}$ of the supernova remnants 65.7+1.2 (DA 495), G67.7+1.8, G69.0+2.7 (CTB 80), G73.9+0.9, G74.9+1.2 (CTB 87), G76.9+1.0, G89.0+4.7 (HB 21), G93.7-0.2 (CTB 104A, DA 551), and G106.3+2.7. The contours indicate the total power emission.

the aforementioned thermal background dominates, and one cannot extract a reasonable flux for the SNR alone due to the low resolution of the data.

\section{G84.2-0.8}

G84.2-0.8 appears to be a compact, elliptical shell-type structure, with the elongated axis $\left(24^{\prime}\right)$ aligned with Galactic longitude (Fig. 3). No significant polarized emission is detected (see Table 4) indicating that W 80 is likely acting as a foreground screen. Its spectrum with a spectral index of $\alpha=-0.5$ (Fig. 8) confirms its nature as an adiabatically expanding shelltype SNR.

\section{G84.9+0.5}

This SNR is of small angular size $\left(\sim 8^{\prime}\right.$ diameter) (Fig. 3) and was discovered by Taylor et al. (1992) in a $327 \mathrm{MHz}$ Galactic plane survey with the Westerbork Synthesis Radio Telescope. No polarized emission at $1420 \mathrm{MHz}$ is detected. Although its classification as an SNR is based on an integrated spectral index of -0.42 (fluxes at $327 \mathrm{MHz}$ and $4850 \mathrm{MHz}$ ), one must treat this with caution, as two extragalactic point sources invade the boundary of the object, and undoubtedly contribute substantial flux of their own at low frequencies. The sourceremoved spectrum between 408 and $1420 \mathrm{MHz}$ has a gradient of $\alpha=-0.08 \pm 0.25$, (Fig. 8)), more reminiscent of an $\mathrm{H}$ II region.

\section{G85.4+0.7}

G85.4+0.7 is a supernova remnant that was discovered in the data of the CGPS (Kothes et al. 2001). It is an unusually looking shell-type SNR with two distinctly visible shells on one side and maybe some diffuse emission patches on the other (Fig. 3). The inner shell is believed to be non-thermal and the outer thermal (Kothes et al. 2001), which might be reflected in its rather flat radio spectrum (Fig. 8). The CGPS data do not reveal any detected polarized emission, likely due to confusion with emission from the radio source $\mathrm{W} 80$.

\section{G85.9-0.6}

G85.9-0.6 (Fig. 3) like G85.4+0.7 was discovered in the data of the CGPS (Kothes et al. 2001). It is considered to be a pure 

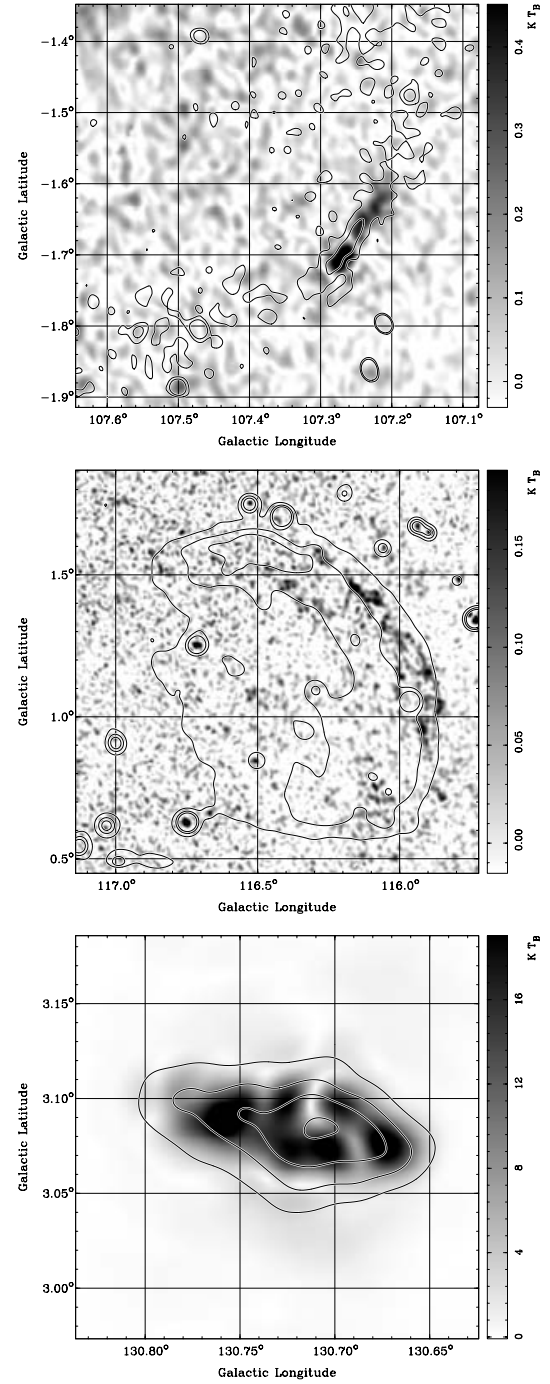
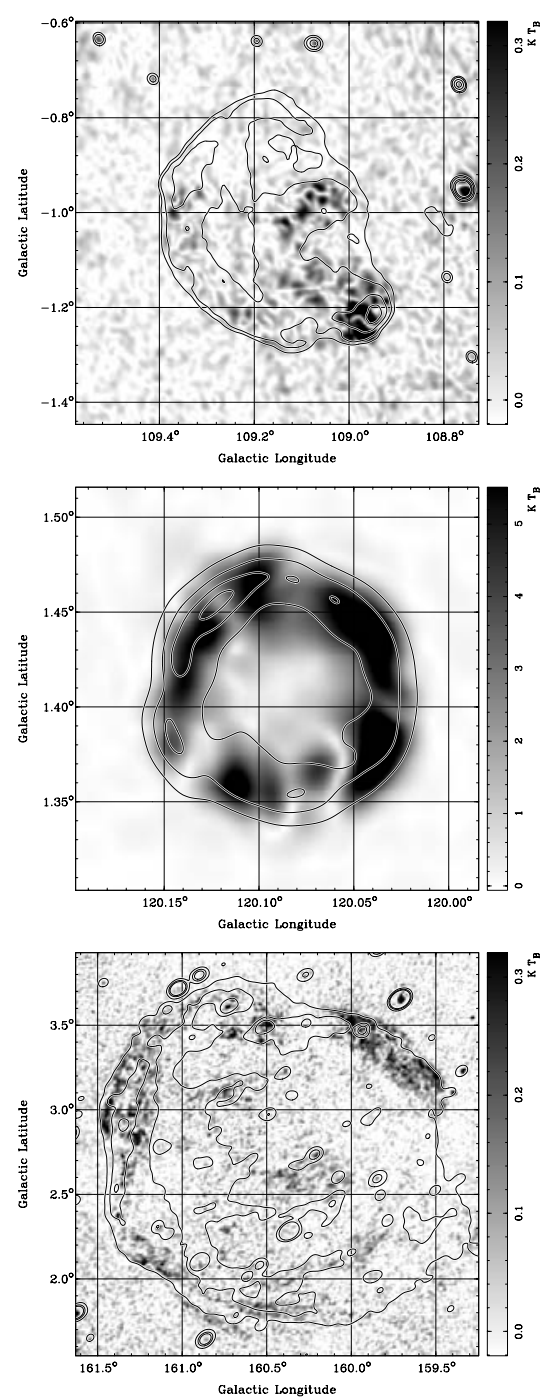
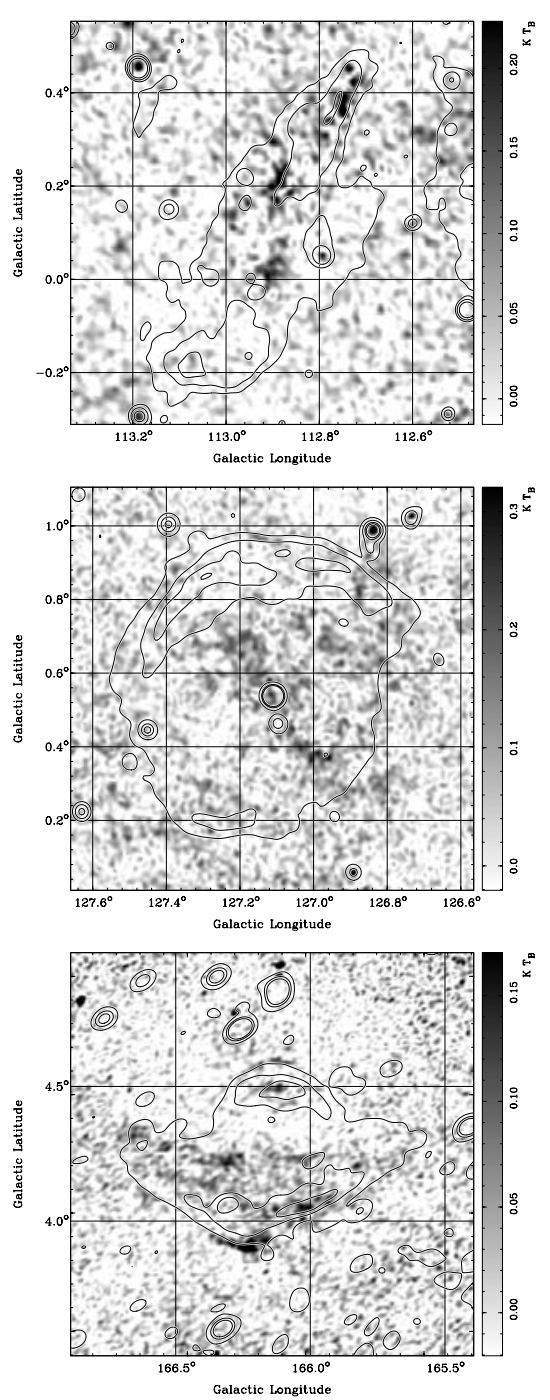

Fig. 13. Images of polarized intensity at $1420 \mathrm{MHz}$ of the supernova remnants G107.5-1.5, G109.1-1.0 (CTB 109), G113.0+2.0, G116.5+1.1, G120.1+1.4 (Tycho, 3C 10, SN 1572), G127.1+0.5, G130.7+3.1 (3C 58, SN 1181), G160.9+2.6 (HB 9), and G166.0+4.3 (VRO 42.05.01). The contours indicate the total power emission.

shell-type remnant, consisting of a faint arc of diameter $\sim 0.4^{\circ}$ centered at $\ell=85.9$ and $b=-0.6$ to the west. The SNR's emission is confused by a bright extragalactic point source in the middle of the arc and diffuse emission from the W 80 region. It is quite difficult to separate its emission from the bright background which is reflected in the high uncertainty in its radio spectrum (Fig. 8). We did not detect any significant polarized emission in the CGPS data, perhaps due to confusion with the radio source $\mathrm{W} 80$.

\section{G89.0+4.7 (HB 21)}

HB 21 is a nearby, large $\left(2.5^{\circ} \times 2.3^{\circ}\right)$ intricately detailed SNR in CGPS images (Fig. 3). With all background sources removed, the spectrum is somewhat flat (Fig. 8), suggesting that much of the radio remnant shows portions evolving through the later stages of the Sedov phase, or early into the radiative (where a significant amount of the shock energy is being radiated away). The SNR shell has sharp boundaries as seen in the $1420 \mathrm{MHz}$ image (Fig. 3), and shows bright polarized emission (Fig. 12) with an average $4 \%$ percentage polarization (peak of $34 \%$, see
Table 4). We likely underestimate the total polarized emission due to missing short spacings.

\section{G93.3+6.9 (DA 530)}

DA 530 is considered to be the picture book example of a typical adiabatically expanding shell-type supernova remnant. In the CGPS this is reflected in its nice double shell structure (Fig. 3) and its radio spectrum with a spectral index of $\alpha=-0.45$ (Fig. 8). Since the $1420 \mathrm{MHz}$ data only covers the Galactic plane up to a latitude of $5.5^{\circ}$ we have no $1420 \mathrm{MHz}$ observations. The position of its centre and its dimension (Table 1) were determined from our $408 \mathrm{MHz}$ data.

\section{G93.7-0.2 (CTB 104A, DA 551)}

CTB 104A is considered to be a pure shell-type SNR. This SNR has already been studied based on CGPS radio continuum and H I data by Uyanıker et al. (2002). It has a rather thick shell (Fig. 4) with no sharp outer boundary and a few smooth extensions to the north and to the south. This might explain the large scatter in its radio continuum spectrum (Fig. 8). CTB 104A is 
Table 4. Polarization properties of the supernova remnants. The polarized intensity was corrected for noise bias (see text Sect. 3.2). The peak polarization in Col. 4 is an approximate value. SNRs with no detectable polarization were removed from the table.

\begin{tabular}{lccc}
\hline \hline Source & PI $_{1420}[\mathrm{mJy}]$ & $\%-$ Pol. & Peak \%-Pol. \\
\hline G65.7+1.2 & $441 \pm 14$ & $11.0 \pm 0.7$ & 26 \\
G67.7+1.8 & $49 \pm 2$ & $7.2 \pm 0.5$ & 50 \\
G69.0+2.7 & $4230 \pm 130$ & $7.6 \pm 0.7$ & 48 \\
G73.9+0.9 & $99 \pm 4$ & $1.3 \pm 0.1$ & 9 \\
G74.9+1.2 & $117 \pm 4$ & $1.6 \pm 0.3$ & 5 \\
G76.9+1.0 & $8 \pm 1$ & $0.6 \pm 0.1$ & 3 \\
G78.2+2.1 & $970 \pm 50$ & $0.4 \pm 0.1$ & 2 \\
G82.2+5.3 & $210 \pm 10$ & $0.23 \pm 0.02$ & 14 \\
G84.2-0.8 & $14 \pm 1$ & $0.19 \pm 0.03$ & 13 \\
G89.0+4.7 & $6690 \pm 750$ & $3.7 \pm 0.4$ & 34 \\
G93.7-0.2 & $5240 \pm 180$ & $15.0 \pm 1.8$ & 43 \\
G106.3+2.7 & $444 \pm 16$ & $9.3 \pm 1.0$ & 41 \\
G107.5-1.5 & $32 \pm 4$ & $46 \pm 7$ & 71 \\
G109.1-1.0 & $210 \pm 12$ & $1.2 \pm 0.1$ & 8 \\
G113.0+0.2 & $125 \pm 7$ & - & 50 \\
G114.3+0.3 & $108 \pm 4$ & $2.0 \pm 0.3$ & 31 \\
G116.5+1.1 & $210 \pm 30$ & $2.0 \pm 0.3$ & 50 \\
G116.9+0.2 & $30 \pm 3$ & $0.4 \pm 0.1$ & 35 \\
G120.1+1.4 & $1010 \pm 30$ & $2.5 \pm 0.1$ & 6 \\
G126.2+1.6 & $52 \pm 7$ & $0.8 \pm 0.2$ & 80 \\
G127.1+0.5 & $640 \pm 70$ & $6.6 \pm 0.8$ & 49 \\
G130.7+3.1 & $1620 \pm 100$ & $5.1 \pm 0.4$ & 10 \\
G160.9+2.6 & $4900 \pm 170$ & $9.1 \pm 0.6$ & 59 \\
G166.0+4.3 & $440 \pm 50$ & $8.6 \pm 1.2$ & 57 \\
\hline & & &
\end{tabular}

highly polarized in the CGPS data (Fig. 12). Because of its large angular size the total polarized emission listed in Table 4 is likely a lower limit due to missing short spacings.

\section{G94.0+1.0 (3C 434.1)}

This Perseus arm object has already been studied extensively based on CGPS radio continuum (Foster 2005) data, and is a textbook example of an SNR evolving within a stellar wind bubble. The SNR is expanding freely in a westerly direction into the bubble's interior, but the shock's energy is being thermalized where it has impacted the inside wall of the SWB (eastern half of 3C 434.1, see Fig. 4). Its overall radio spectrum has an index $\alpha=-0.48$ (Fig. 9), typical of an mature shell-type remnant. No $1420 \mathrm{MHz}$ polarized emission is observed in the CGPS.

\section{G96.0+2.0}

G96.0+2.0 was discovered in the data of the Canadian Galactic Plane Survey (Kothes et al. 2005) and is believed to be a pure shell-type remnant expanding inside a stellar wind bubble. It consists of a shell to the west and diffuse emission slowly fading away to the east (Fig. 4). The SNR has a typical radio spectrum with an index of $\alpha=-0.45$ (Fig. 9) indicating an adiabatically expanding shell-type SNR. Like 3C 434.1, it is unpolarized in the CGPS data, likely the result of a large distance, and perhaps internal effects.

\section{G106.3+2.7}

A previous study based on CGPS data (Kothes et al. 2001) found that the combined type supernova remnant G106.3+2.7 consists of a diffuse shell-type remnant breaking out to the west into the interior of a stellar wind bubble and a small pulsar wind nebula sitting at the top of a somewhat brighter head structure to the east (Fig. 4). Its radio spectrum with $\alpha=-0.61$ (Fig. 9) is somewhat steep, especially considering that this remnant contains a PWN, which have flatter spectra; however, the shell-type remnant is certainly the dominating source of the radio continuum emission and the breakout into the interior of the stellar wind bubble might give the remnant characteristics of an early stage of evolution. G106.3+2.7 is highly polarized in the $1420 \mathrm{MHz}$ CGPS data (Fig. 12).

\section{G107.5-1.5}

G107.5-1.5 was discovered with data from the Canadian Galactic Plane Survey (Kothes 2003). It consists of a short narrow shell-like feature (Fig. 4). Its shape and radio spectrum with an index of $\alpha=-0.56$ (Fig. 9) suggest an adiabatically expanding shell-type SNR. G107.5-1.5 is with 50\% integrated polarization the source with the highest percentage polarization in the whole CGPS (Fig. 13). This is likely the result of low foreground depolarization.

\section{G109.1-1.0 (CTB 109)}

The SNR CTB 109 was identified as an SNR by Gregory \& Fahlman (1980) on the basis of X-ray data observed with the Einstein Satellite. In the CGPS the SNR has a peculiar semicircular shape (Fig. 4) and its radio spectrum has an index of $\alpha=-0.50$ (Fig. 9), typical for a mature shell-type SNR. There is weak polarization coming from this SNR (Fig. 13), but curiously it is confined to the area below about $-0.9^{\circ}$ of Galactic Latitude, probably the result of depolarization by a foreground cloud of ionized gas that is partly overlapping with the SNR.

\section{G113.0+0.2}

G113.0+0.2 was discovered as an SNR with data from the CGPS (Kothes et al. 2005). It is highly linearly polarized at $1420 \mathrm{MHz}$ (see Fig. 13), and has an unusual elongated appearance consisting of a long polarized filament and a complex head structure (Fig. 5), that is interacting with a small molecular cloud. Due to confusion with background emission no reliable flux density can be extracted at $1420 \mathrm{MHz}$. At $408 \mathrm{MHz}$, image artifacts from nearby SNR Cas A cause additional problems.

\section{G114.3+0.3}

The SNR G114.3+0.3 consists of a thin shell to the west with a sharp outer boundary sitting on top of diffuse emission (Fig. 5). The image quality at $408 \mathrm{MHz}$ is very poor due to artifacts from nearby SNR Cas A so that we could not publish a reliable flux density at that frequency. This SNR has been studied in detail with data from the CGPS by Yar-Uyanıker et al. (2004) and Tian \& Leahy (2006b). Its shell-like appearance and radio spectrum ( $\alpha=-0.49$, Fig. 9) suggests it is an adiabatically expanding shell-type SNR. The SNR is only weakly polarized in our data (Table 4), which could be the result of its very low surface brightness in total power.

\section{G116.5+1.1}

The SNR G116.5+1.1 appears as a thick shelled SNR in our $1420 \mathrm{MHz}$ data (Fig. 5). It has the typical radio spectrum of an adiabatically expanding shell-type SNR, with $\alpha=-0.53$ 
(Fig. 9). This SNR has been studied in detail with data from the CGPS by Yar-Uyanıker et al. (2004) and Tian \& Leahy (2006b). The SNR is weakly polarized in our $1420 \mathrm{MHz}$ data (Fig. 13).

\section{G116.9+0.2 (CTB 1)}

CTB 1 is a well studied shell-type supernova remnant. In our $1420 \mathrm{MHz}$ image (Fig. 5) it consists of two well defined shells to the west and the south-east and an opening to the north-east. The radio spectrum with a spectral index of $\alpha=-0.61$ (Fig. 9) is somewhat steep for a typical adiabatically expanding shelltype SNR. The integrated percentage polarization is with $0.5 \%$ extremely low (Table 4). The reason for this is still unclear, but could be the result of strong internal effects. This SNR has been studied with data from the CGPS by Yar-Uyanıker et al. (2004) and Tian \& Leahy (2006b).

\section{G120.1+1.4 (Tycho, 3C 10, SN 1572)}

The remnant of Tycho's SN is a circular ring of $10.8^{\prime}$ diameter in the CGPS $1420 \mathrm{MHz}$ images (Fig. 5), of total flux density $S_{v=1420}=40.5 \pm 1.5 \mathrm{Jy}$. It is the only proven remnant of a type Ia SN in the CGPS area. Its spectrum (Fig. 9) declines very constantly at higher frequencies, with $\alpha=-0.65 \pm 0.01(-0.61$ between CGPS flux points), typical for a young shell-type SNR in the pre-adiabatic expansion phase. Tycho is $2.5 \%$ polarized at $1420 \mathrm{MHz}$ (Fig. 13, Table 4).

\section{G126.2+1.6}

G126.2+1.6 has been studied with radio continuum data from the CGPS by Tian \& Leahy (2006a). It is a large $\left(1.5^{\circ} \times 1.4^{\circ}\right)$ very diffuse remnant in the CGPS, resolved into fragmented filaments at $1420 \mathrm{MHz}$ (Fig. 5), and nearly impossible to determine a reliable $408 \mathrm{MHz}$ flux density for. This is likely the reason why our $408 \mathrm{MHz}$ flux density differs significantly from the one published by Tian \& Leahy (2006a), which is based on the same data. The continuum appearance has a sharp shell boundary, especially in the West. A brightened $(0.4 \mathrm{~K}$ above the mean background in the remnant's interior), diffuse circular patch of emission about $7^{\prime}$ in radius is found at the remnant's centre. It may suggest the presence of a pulsar-wind nebula, but clearly more observations are needed to confirm this speculation. Over the entire SNR, emission is weakly polarized $(0.8 \%)$, but one dim continuum filament in the west $\left(\ell=125.6^{\circ}, b=+1.57^{\circ}\right)$ is remarkably $80 \%$ polarized (Table 4$)$.

\section{G127.1+0.5 (R 5)}

G127.1+0.5 is a very apparent shell-type SNR at $1420 \mathrm{MHz}$, much like G116.9+0.2 in appearance (Fig. 6). A bright $(80 \mathrm{~K}$ peak at $1420 \mathrm{MHz}$ ) extra-galactic source also sits in the bull'seye of the SNR. The SNR's spectrum is fairly well defined by 10 flux density points (see Fig. 10), and the gradient $\alpha=$ $-0.45 \pm 0.02$ is typical of shell-type supernova remnants. The $1420 \mathrm{MHz}$ emission from G127.1+0.5 is $6.6 \%$ polarized, peaking at $49 \%$ (Fig. 13).

\section{G130.7+3.1 (3C 58, SN 1181)}

This remnant originates with SN 1181, and is a flat-spectrum ( $\alpha=-0.07 \pm 0.01$, see Fig. 10$)$ and strongly polarized $(5.1 \%$ integrated) pulsar wind nebula (Fig. 13). In $1420 \mathrm{MHz}$ CGPS data, 3C 58 is a rather featureless, filled-centre source elongated along Galactic longitude $\left(11^{\prime} \times 6^{\prime}\right)$ (Fig. 6).

\section{G132.7+1.3 (HB 3)}

HB 3 has been studied with radio continuum data from the CGPS by Tian \& Leahy (2005). Although this shell-type SNR is partially confused by thermal emission from W3 (Fig. 6), it has a quite straight and steep spectrum with $\alpha=-0.66$ (Fig. 10). It is not visible in polarized emission probably the result of the confusion with $\mathrm{W} 3$. The dimensions determined here (Table 1) show that HB 3 is one of the largest SNRs in our sample and thus presumably of quite an advanced age. The steepness of its radio spectrum (Fig. 10) is remarkable for an advanced remnant.

\section{G156.2+5.7}

G156.2+5.7 is a quite unique object since it is in X-rays one of the brightest SNRs known; it was discovered in the ROSAT all-sky survey (Pfeffermann et al. 1991). However, it is the SNR with the lowest radio surface brightness observed to date (Reich et al. 1992). This is the reason why we had to remove the point sources and then convolve the $408 \mathrm{MHz}$ image to $5^{\prime}$ to make the remnant apparent (Fig. 6). Our $408 \mathrm{MHz}$ flux density nicely confirms its non-thermal radio spectrum with a spectral index of $\alpha=-0.53$ (Fig. 10), typical for an adiabatically expanding shelltype SNR. Since the $1420 \mathrm{MHz}$ data only covers the Galactic plane up to a latitude of $+5.5^{\circ}$ we have no $1420 \mathrm{MHz}$ observations. The position of its centre and its dimension (Table 1) were determined from our $408 \mathrm{MHz}$ data.

\section{G160.9+2.6 (HB 9)}

This anticentre SNR joins HB 3 as enigmatic, with its large extent on the sky $\left(2.48^{\circ} \times 2.32^{\circ}\right)$ and steep radio spectrum $(\alpha=-0.64 \pm 0.02)$. By excluding point sources, we obtain $\alpha=-0.51 \pm 0.07$ between 408 and $1420 \mathrm{MHz}$, somewhat flatter than the multi-wavelength spectrum in Fig. 10 (where most fluxes include background sources). The continuum appearance is that of a multitude of fragmented shells overlapping one another (Fig. 6). It does seem clear that this is a very large, evolved remnant, which is probably cooling rapidly due to radiative losses (e.g. the optical emission). A high degree of linear polarization $(9 \%$ at $1420 \mathrm{MHz}$ ) is seen for the whole remnant, peaking at $59 \%$ (Fig. 13). The polarized emission is mainly confined to the outer filaments where continuum emission is strongest.

\section{G166.0+4.3 (VRO 42.05.01)}

VRO 42.05.01 is an unusually-shaped SNR, showing two shells of strikingly different radii. The $1420 \mathrm{MHz}$ image (see Fig. 6) gives the appearance of a remnant evolving into two media of unequal density. We find the entire remnant has an integrated spectral index of $\alpha=-0.37 \pm 0.11$ (Fig. 10). Leahy \& Tian (2005) find based on CGPS data that the northern "shell region" has $\alpha=-0.31 \pm 0.03$, while the southern "wing region" has $\alpha=-0.47 \pm 0.03^{2}$, also suggestive that two different media are modifying the shock. This SNR is quite polarized, showing $8.6 \%$ linearly polarized emission (more so in the "wing"region), peaking at $57 \%$ (Fig. 13).

\footnotetext{
2 These are all point- source corrected values.
} 


\section{Summary}

This catalogue presents radio continuum and polarization properties of all known supernova remnants observed in the CGPS $\left(65^{\circ} \leq \ell \leq 175^{\circ},-5^{\circ} \leq b \leq+7^{\circ}\right)$. Our sample was initially taken from the SNR catalogue of Green (2004). We have amended this sample somewhat to include two new confirmed SNRs (G83.0-0.3, G107.5-1.5). Additionally, nine other SNRs listed by Green (2004) as "possible" were investigated. G71.6-0.5, G72.2-0.3, and G85.2-1.2 (Taylor et al. 1992), as well as G75.5+2.4 are inconclusive with the current observations. G104.7+2.8 and G74.8+0.63 have measured thermal radio spectra, and are most likely $\mathrm{H}$ II regions. Three possibilities (G69.4+1.2, G70.0+2.0 and G117.7+0.6) simply do not appear in CGPS images. Finally, the newly discovered H II region OA 184 (Foster et al. 2006) is not included in our list.

Flux densities, spectral indices and fractional polarization are measured for all SNRs that remained. The current list consists of 36 SNRs, and is reasonably "complete" in the sense that all SNRs known and discovered at the time of this writing (February 2006) and within the bounds of the CGPS are presented. Certainly, the current sample does not represent the total population of remnants in the CGPS region, due to limitations in the survey's sensitivity. As well, at least three unconfirmed new candidates remain in the CGPS (as suspected by the authors), but are not yet presentable at the time of this writing.

This catalogue is useful as a reference for SNRs, and will provide a comprehensive starting point for the researcher wishing to study individual or group statistics of SNRs within the CGPS region. To this end, we give mosaic designations for CGPS data (Table 1; FITS files for each can be obtained from the CADC at http://cadc-ccda.hia-iha.nrc-cnrc.gc.ca/ cgps.

Acknowledgements. We wish to thank Dave Routledge (University of Alberta) for careful reading of the manuscript. We also wish to thank the anonymous referee for comments which improved this paper. The Dominion Radio Astrophysical Observatory is a National Facility operated by the Nationa Research Council. The Canadian Galactic Plane Survey is a Canadian project with international partners, and is supported by the Natural Sciences and Engineering Research Council (NSERC).

\section{References}

Albinson, J. S., Tuffs, R. J., Swinbank, E., \& Gull, S. F. 1986, MNRAS, 219, 427 Angerhofer, P. E., Kundu, M. R., \& Becker, R. H. 1977, A\&A, 55, 11

Chevalier, R. A., Kirshner, R. P., \& Raymond, J. C. 1980, ApJ, 235, 186 Condon, J. J., Broderick, J. J., \& \& Seielstad, G. A. 1989, AJ, 97, 1064 Condon, J. J., Cotton, W. D., Greisen, E. W., et al. 1998, AJ, 115, 1693 Dickel, J. R., \& DeNoyer, L. K. 1975, AJ, 80, 437 Downes, A. 1983, MNRAS, 203, 695

Dwarakanath, K. S., Shevgaonkar, R. K., \& Sastry, Ch. V. 1982, JApA, 3, 207 Foster, T. 2005, A\&A, 441, 1043

Foster, T., Kothes, R., Sun, X. H., Reich, W., \& Han, J. L. 2006, A\&A, 454, 517 Fürst, E., Reich, W., Reich, P., \& Reif, K. 1990, A\&AS, 85, 691

Goss, W. M., Mantovani, F., Salter, C. J., Tomasi, P., \& Velusamy, T. 1984, A\&A, 138,469

Green, A. J., Baker, J. R., \& Landecker, T. L. 1975, A\&A, 44, 187

Green, D. 1986, MNRAS, 218, 533

Green, D. A. 2004, Bull. Astron. Soc. India, 32, 335 (also available http://www.mrao.cam.ac.uk/surveys/snrs)

Green, D. A, \& Joncas, G. 1994, A\&AS, 104, 481

Gregory, P. C., \& Fahlman, G. G. 1980, Nature, 287, 805

Hailey, C. J., \& Craig, W. W. 1995, ApJ, 455, L151
Haslam, C. G. T., Salter, C. J., \& Pauls, T. 1980, A\&A, 92, 57

Haslam, C. G. T., Stoffel, H., Salter, C. J., \& Wilson, W. E. 1982, A\&AS, 47, 1

Higgs, L. A., Landecker, T. L., \& Roger, R. S. 1977, AJ, 82, 718

Higgs, L. A., Landecker, T. L., Israel, F. P., \& Bally, J. 1991, JRASC, 85, 24

Hirabayashi, H., \& Takahashi, T. 1972, PASJ, 24, 231

Hughes, V. A., Harten, R. H., \& van den Bergh, S. 1981, ApJ, 246, L127

Hughes, V. A., Harten, R. H., Costain, C. H., Nelson, L. A., \& Viner, M. R. 1984, ApJ, 283, 147

Joncas, G., Roger, R. S., \& Dewdney, P. E. 1989, A\&A, 219, 303

Klein, U., Emerson, D. T., Haslam, C. G. T., \& Salter, C. J. 1979, A\&A, 76, 120 Kothes, R. 2003, A\&A, 408, 187

Kothes, R., Landecker, T. L., Foster, T., \& Leahy, D. A. 2001, A\&A, 376, 641

Kothes, R., Uyanıker, B., \& Reid, R. 2005, A\&A, 444, 871

Lalitha, P., Salter, C. J., Mantovani, F., \& Tomasi, P. 1984, A\&A, 131, 196

Landecker, T. L., \& Caswell, J. L. 1983, AJ, 88, 1810

Landecker, T. L., Pineault, S., Routledge, D., \& Vaneldik, J. F. 1982a, ApJ, 261, L41

Landecker, T. L., Roger, R. S., \& Dewdney, P. E. 1982b, AJ, 87, 1379

Landecker, T. L., Higgs, L. A., \& Roger, R. S. 1985, AJ, 90, 1082

Landecker, T. L., Dewdney, P. E., Vaneldik, J. F., \& Routledge, D. 1987, AJ, 94, 111

Landecker, T. L., Purton, C. R., \& Clutton-Brock, M. 1990, A\&A, 232, 207

Landecker, T. L., Higgs, L. A., \& Wendker, H. J. 1993, A\&A, 276, 522

Landecker, T. L., Dewdney, P. E., Burgess, T. A., et al. 2000, A\&AS, 145, 509

Leahy, D. A., \& Tian, W. 2005, A\&A, 440, 929

Mantovani, F., Nanni, M., Salter, C. J., \& Tomasi, P. 1982, A\&A, 105, 176

Mantovani, F., Reich, W., Salter, C. J., \& Tomasi, P. 1985, A\&A, 145, 50

Mantovani, F., Tomasi, P., \& Salter, C. J. 1991, A\&A, 247, 545

Matthews, H. E., \& Shaver, P. A. 1980, A\&A, 87, 255

Matthews, H. E., Baars, J. W. M., Wendker, H. J., \& Goss, W. M. 1977, A\&A, 55,1

Mavromatakis, F., Boumis, P., \& Paleologou, E. V. 2002, A\&A, 387, 635

Mavromatakis, F., Boumis, P., Xilouris, E., Papamastorakis, J., \& Alikakos, J. 2005, A\&A, 435, 141

Morsi, H. W., \& Reich, W. 1987, A\&AS, 69, 533

Nichols-Bohlin, J., Fesen, R. A.,

Pauls, T. 1977, A\&A, 59, L13 1993, AJ, 105, 672

Padmanabhan, T. 2001, Theoretical Astrophysics, Vol. II: Stars and Stellar Systems (Cambridge University Press), 21

Pfeffermann, E., Aschenbach, B., \& Predehl, P. 1991, A\&A, 246, L28

Pineault, S., \& Chastenay, P. 1990, MNRAS, 246, 169

Pineault, S., \& Joncas, G. 2000, AJ, 120, 3218

Reich, P., \& Reich, W. 1986, A\&AS, 63, 205

Reich, W. 1982, A\&AS, 48, 219

Reich, W., \& Braunsfurth, E. 1981, A\&A, 99, 17

Reich, W., Kallas, E., \& Steube, R. 1979, A\&A, 78, L13

Reich, W., Fürst, E., Reich, P., Sofue, Y., \& Handa, T. 1986, A\&A, 155, 185

Reich, W., Fürst, E., Reich, P., \& Junkes, N. 1988, Supernova Remnants and the Interstellar Medium, Proceedings of IAU Coll. 101, ed. R. S. Roger, \&

T. L. Landecker (Cambridge: University Press), 293

Reich, W., Reich, P., \& Fürst, E. 1990, A\&AS, 83, 539

Reich, W., Fürst, E., \& Arnal, E. M. 1992, A\&A, 256, 214

Reich, W., Zhang, X., \& Fürst, E. 2003, A\&A, 408, 961

Rengelink, R. B., Tang, Y., de Bruyn, A. G., et al. 1997, A\&AS, 124, 259

Roger, R. S., Costain, C. H., Landecker, T. L., \& Swerdlyk, C. M. 1999, A\&AS, 137,7

Sofue, Y., Takahara, F., \& Hirabayashi, H. 1983, PASJ, 35, 447

Taylor, A. R., Wallace, B. J., \& Goss, W. M. 1992, AJ, 103, 931

Taylor, A. R., Gibson, S. J., Peracaula, M., et al. 2003, AJ, 124, 3145

Tian, W. W., \& Leahy, D. 2005, A\&A, 436, 187

Tian, W. W., \& Leahy, D. 2006a, A\&A, 447, 205

Tian, W. W., \& Leahy, D. 2006b, ChJAA, accepted

Uyanıker, B., Kothes, R., \& Brunt, C. M. 2002, ApJ, 565, 1022

Velusamy, T., \& Kundu, M. R. 1974, A\&A, 32, 375

Vollmer, B., Davoust, E., Dubois, P., et al. 2005, A\&A, 431, 1177

Wardle, J. F. C., \& Kronberg, P. P. 1974, ApJ, 194, 249

Weiler, K. W., \& Shaver, P. A. 1978, A\&A, 70, 389

Wendker, H. J., Higgs, L. A., \& Landecker, T. L. 1991, A\&A, 241, 551

Willis, A. G. 1973, A\&A, 26, 237

Yar-Uyanıker, A., Uyanıker, B., \& Kothes, R. 2004, ApJ, 616, 247

Yoshita, K., Miyata, E., \& Tsunemi, H. 1999, Astron. Nachr., 320, 344

Zhang, X. Z. 2003, Acta Astron. Sinica, 44, 183 
R. Kothes et al.: SNR catalogue. I., Online Material p 1

\section{Online Material}


R. Kothes et al.: SNR catalogue. I., Online Material p 2
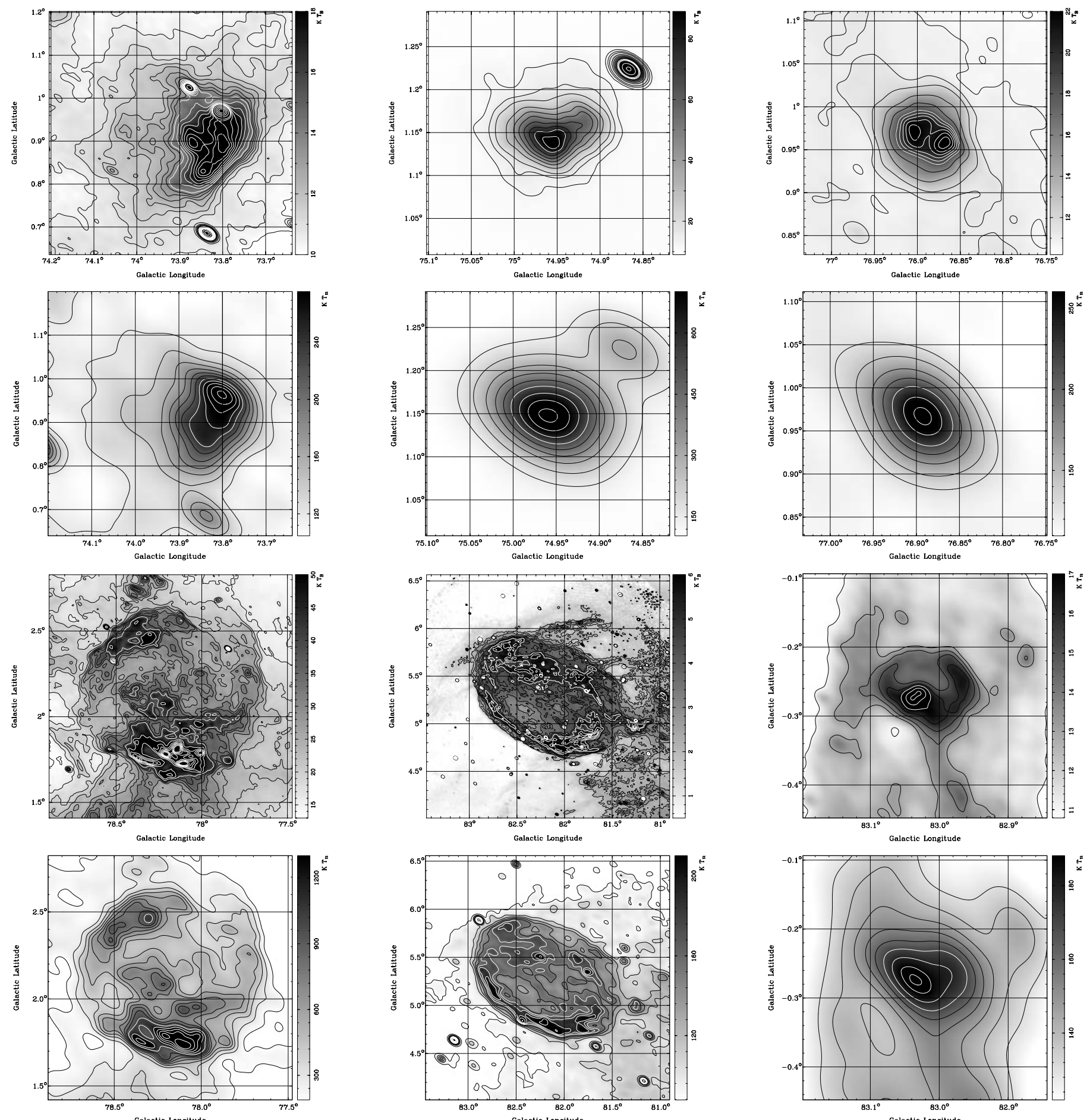

Fig. 2. Total power images at $1420 \mathrm{MHz}$ (first and third row) and at $408 \mathrm{MHz}$ (second and fourth row) of the supernova remnants G73.9+0.9, G74.9+1.2 (CTB 87), G76.9.3+1.0, G78.2+2.1 (DR 4, $\gamma$-Cygni), G82.2+5.3 (W 63), and G83.0-0.3. 
R. Kothes et al.: SNR catalogue. I., Online Material p 3
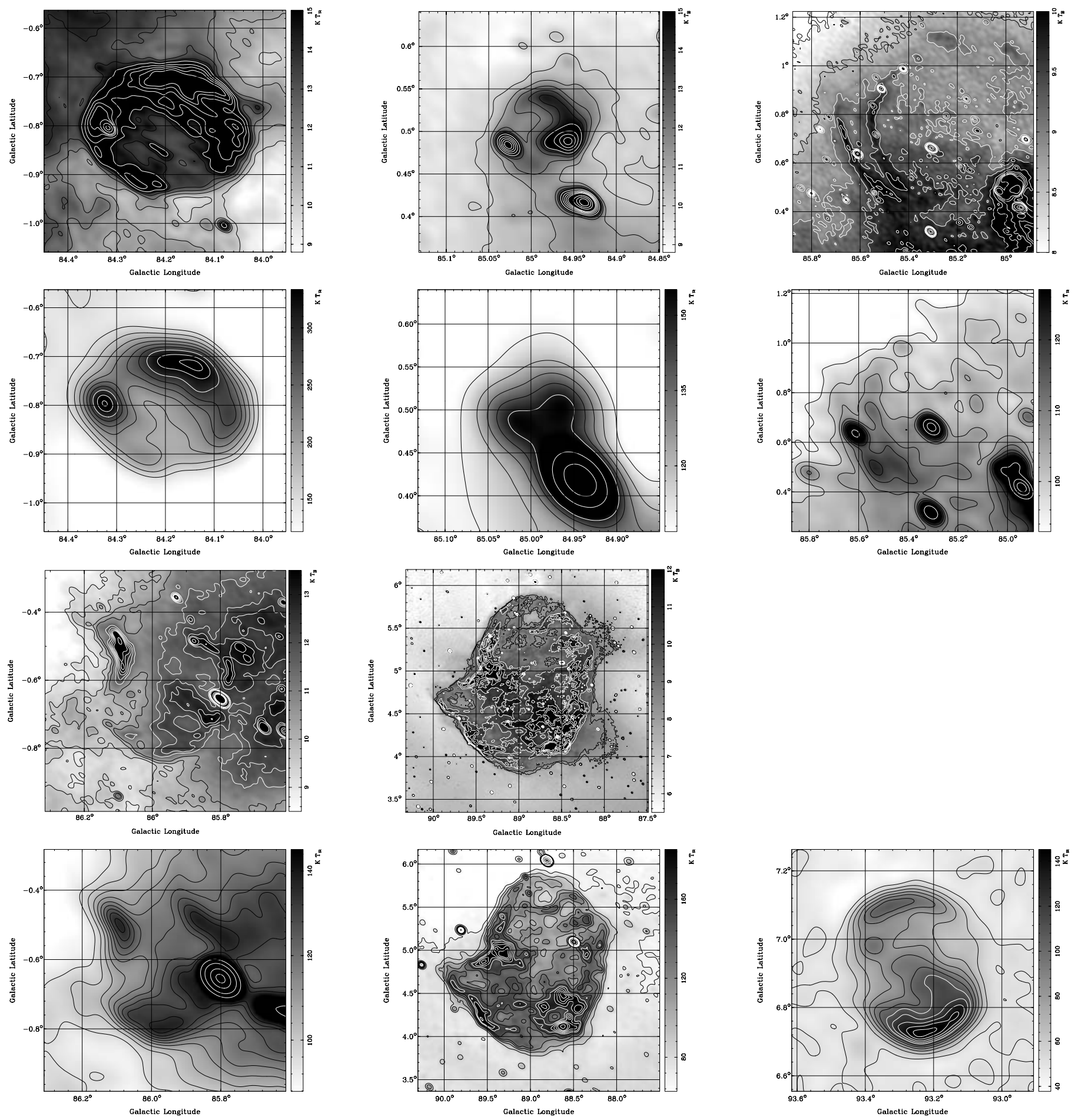

Fig. 3. Total power images at $1420 \mathrm{MHz}$ (first and third row) and $408 \mathrm{MHz}$ (second and fourth row) of the supernova remnants G84.2-0.8, G84.9+0.5, G85.4+0.7, G85.9-0.6, G89.0+4.7 (HB 21), and G93.3+6.9 (DA 530). 
R. Kothes et al.: SNR catalogue. I., Online Material $p 4$
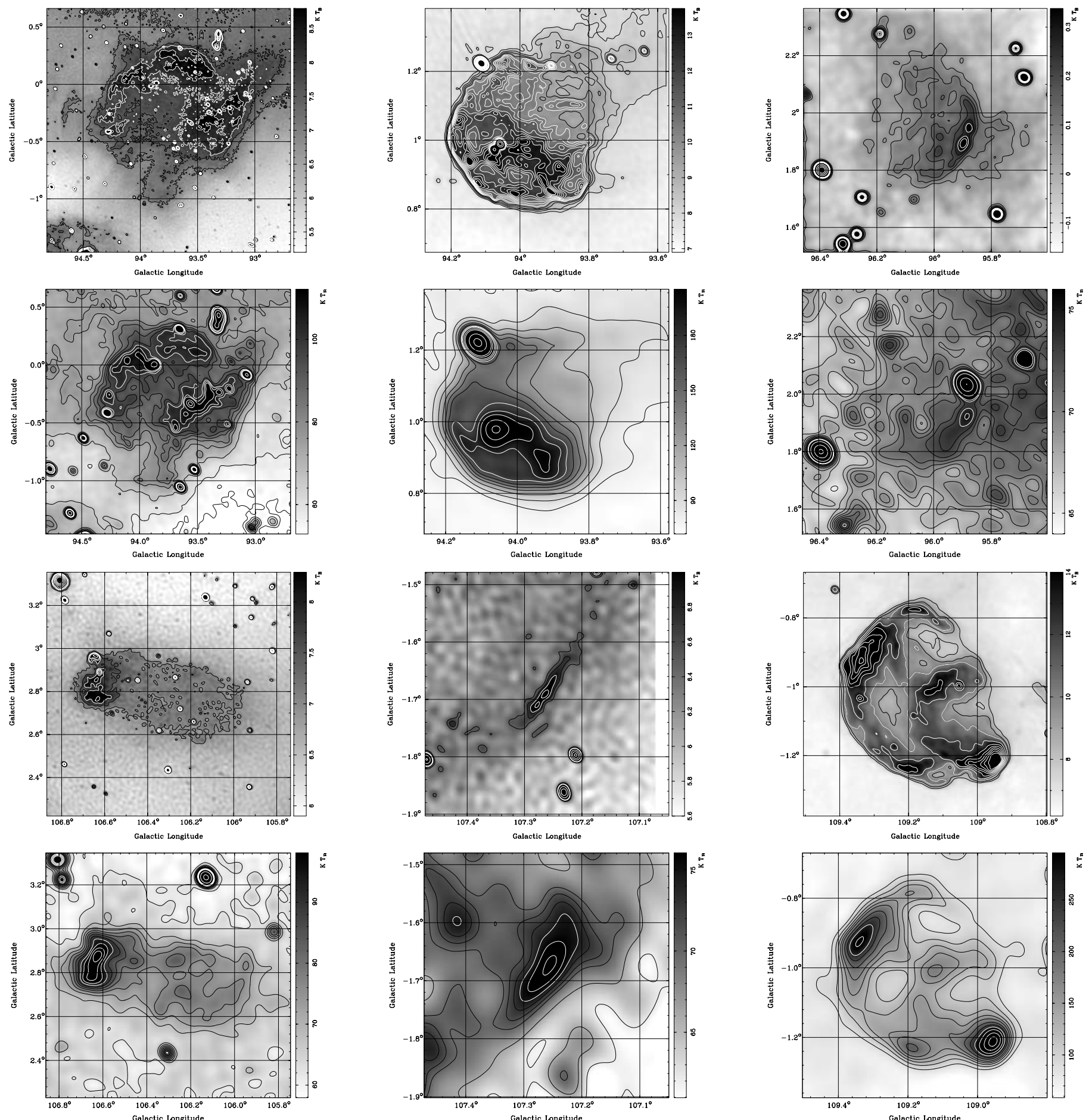

Fig. 4. Total power images at $1420 \mathrm{MHz}$ (first and third row) and $408 \mathrm{MHz}$ (second and fourth row) of the supernova remnants G93.7-0.2 (CTB 104A), G94.0+1.0 (3C 434.1), G96.0+2.0, G106.3+2.7, G107.5-1.5, and G109.1-1.0 (CTB 109). 
R. Kothes et al.: SNR catalogue. I., Online Material p 5
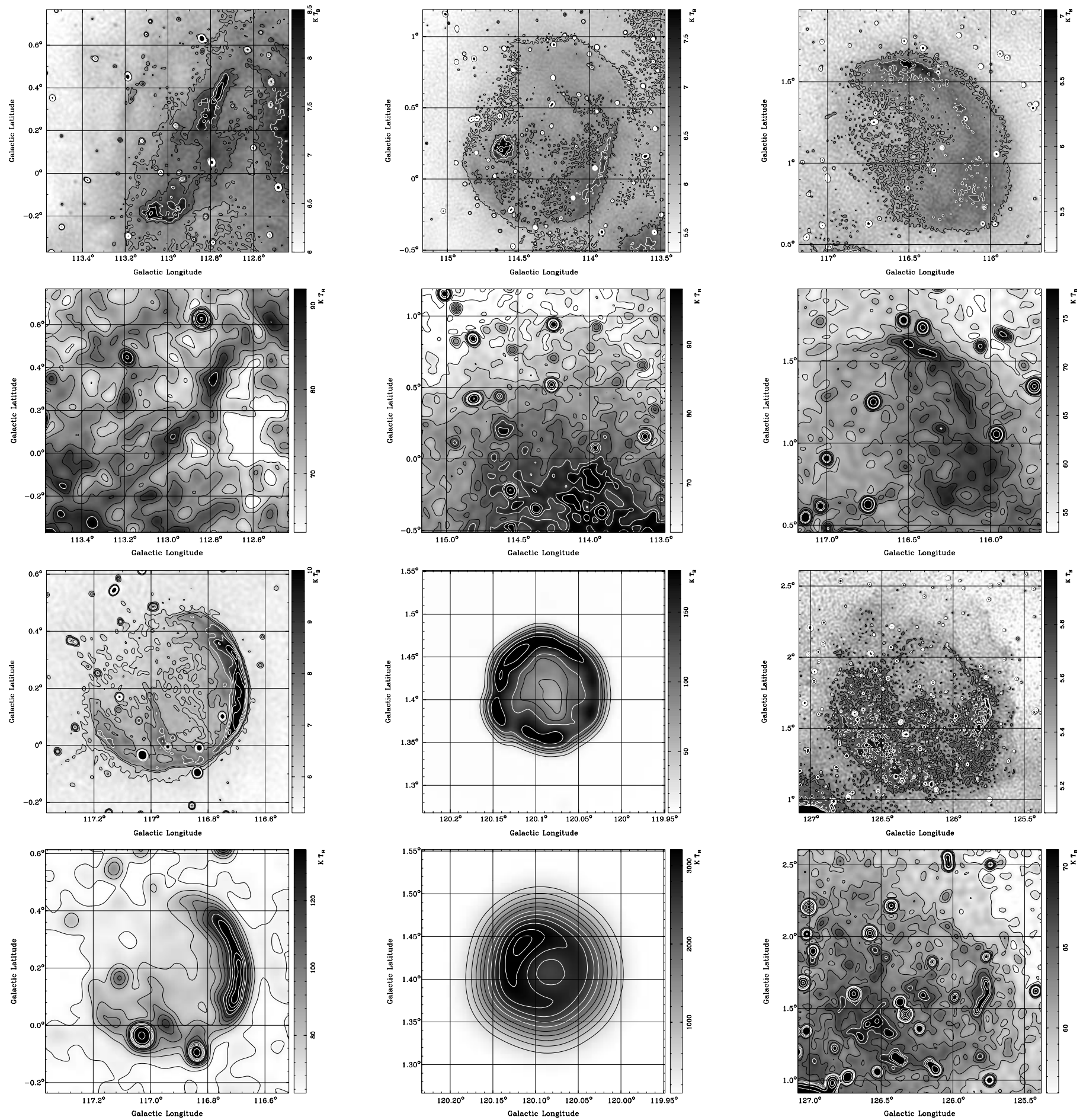

Fig. 5. Total power images at $1420 \mathrm{MHz}$ (first and third row) and $408 \mathrm{MHz}$ (second and fourth row) of the supernova remnants G113.0+0.1, G114.3+0.3, G116.5+1.1, G116.9+0.2 (CTB 1), G120.1+1.4 (Tycho's SNR), and G126.2+1.6. 
R. Kothes et al.: SNR catalogue. I., Online Material p 6
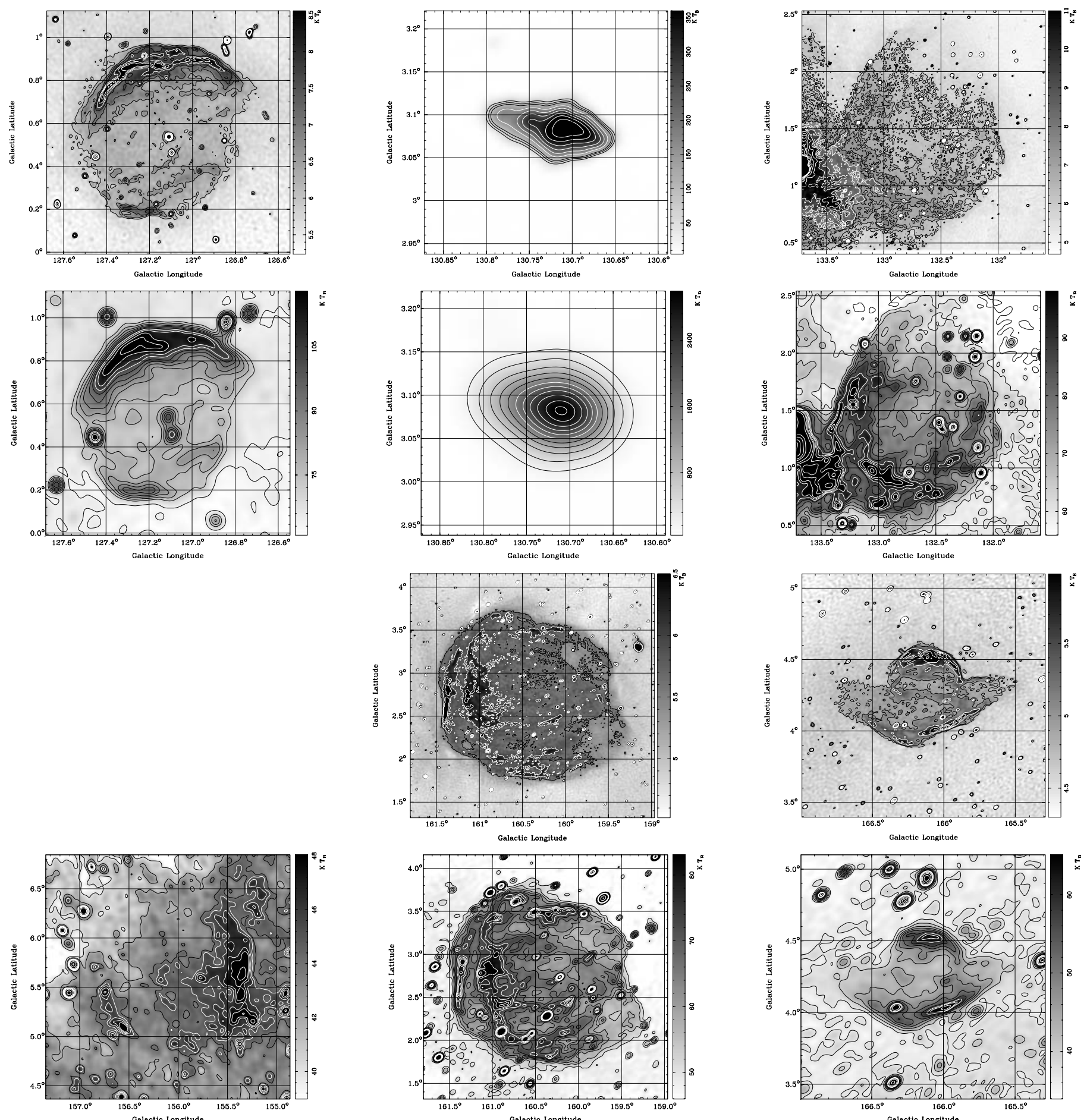

Fig. 6. Total power images at $1420 \mathrm{MHz}$ (first and third row) and $408 \mathrm{MHz}$ (second and fourth row) of the supernova remnants G127.1+0.5, G130.7+3.1 (3C 58), G132.7+1.3 (HB 3), G156.2+5.7, G160.9+2.6 (HB 9), and G166.0+4.3 (VRO 42.05.01). 
R. Kothes et al.: SNR catalogue. I., Online Material $p 7$

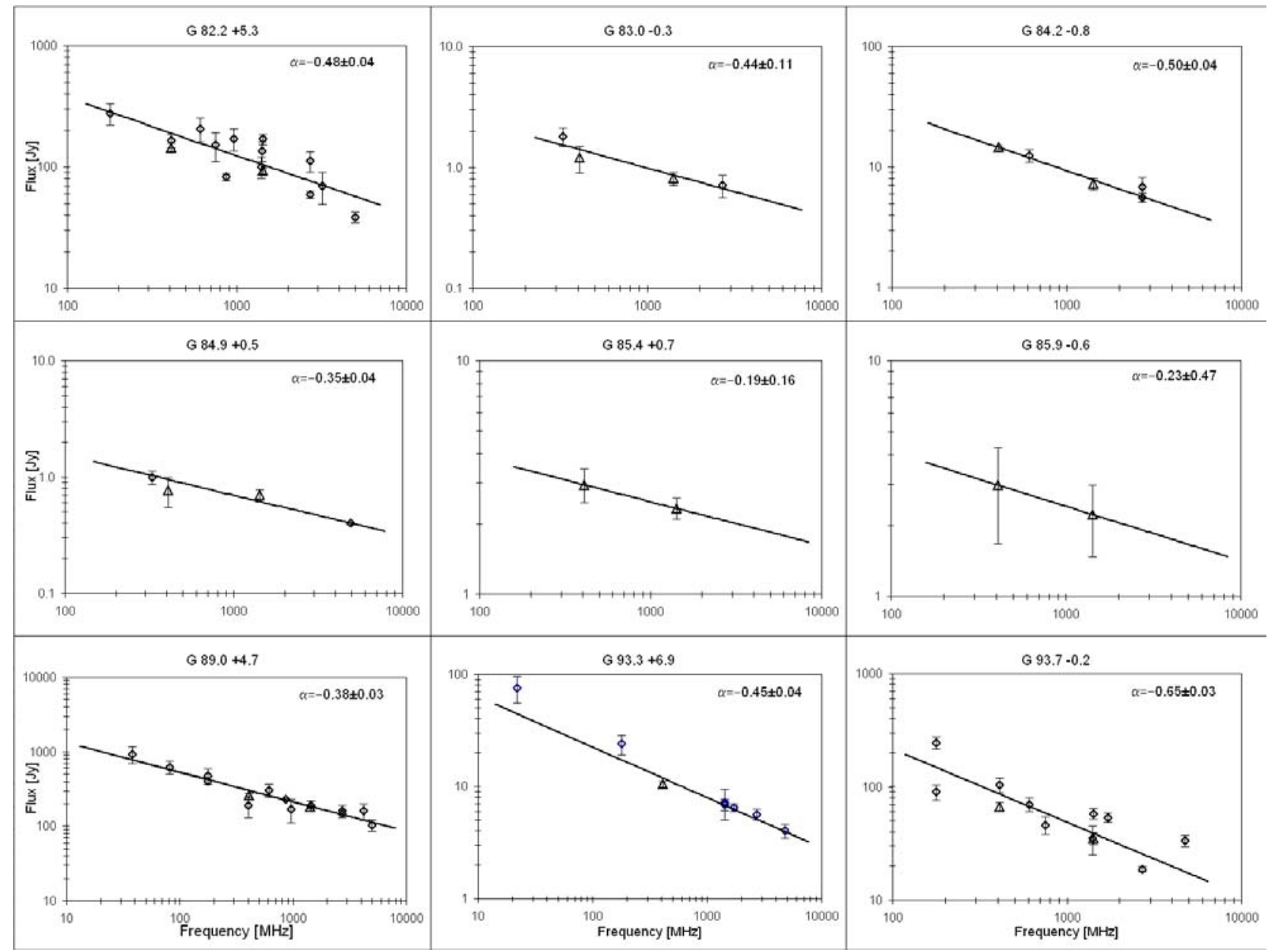

Fig. 8. The radio continuum spectra of the SNRs G82.2+5.3 (W 63), G83.0-0.3, G84.2-0.8, G84.9+0.5, G85.4+0.7, G85.9-0.6, G89.0+4.7 (HB 21), G93.3+6.9 (DA 530), and G93.7-0.2 (CTB 104A, DA 551). The CGPS flux densities are represented by triangles. 
R. Kothes et al.: SNR catalogue. I., Online Material $p 8$

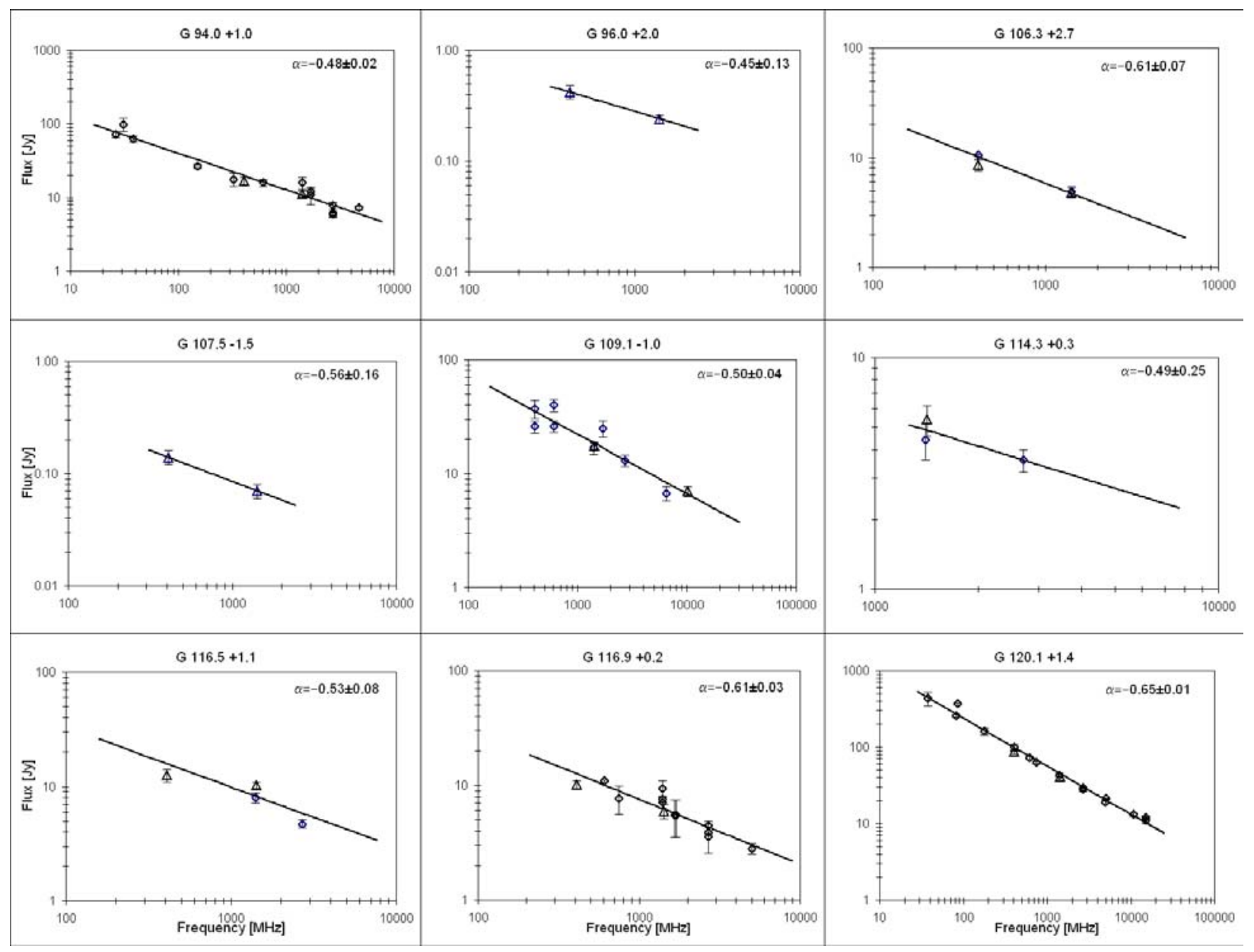

Fig. 9. The radio continuum spectra of the SNRs G94.0+1.0, G96.0+2.0, G106.3+2.7, G107.5-1.5, G109.1-1.0 (CTB 109), G114.3+0.3, G116.5+1.1, G116.9+0.2 (CTB 1), and G120.1+1.4 (Tycho, 3C 10, SN 1572). The CGPS flux densities are represented by triangles. 
R. Kothes et al.: SNR catalogue. I., Online Material $p 9$

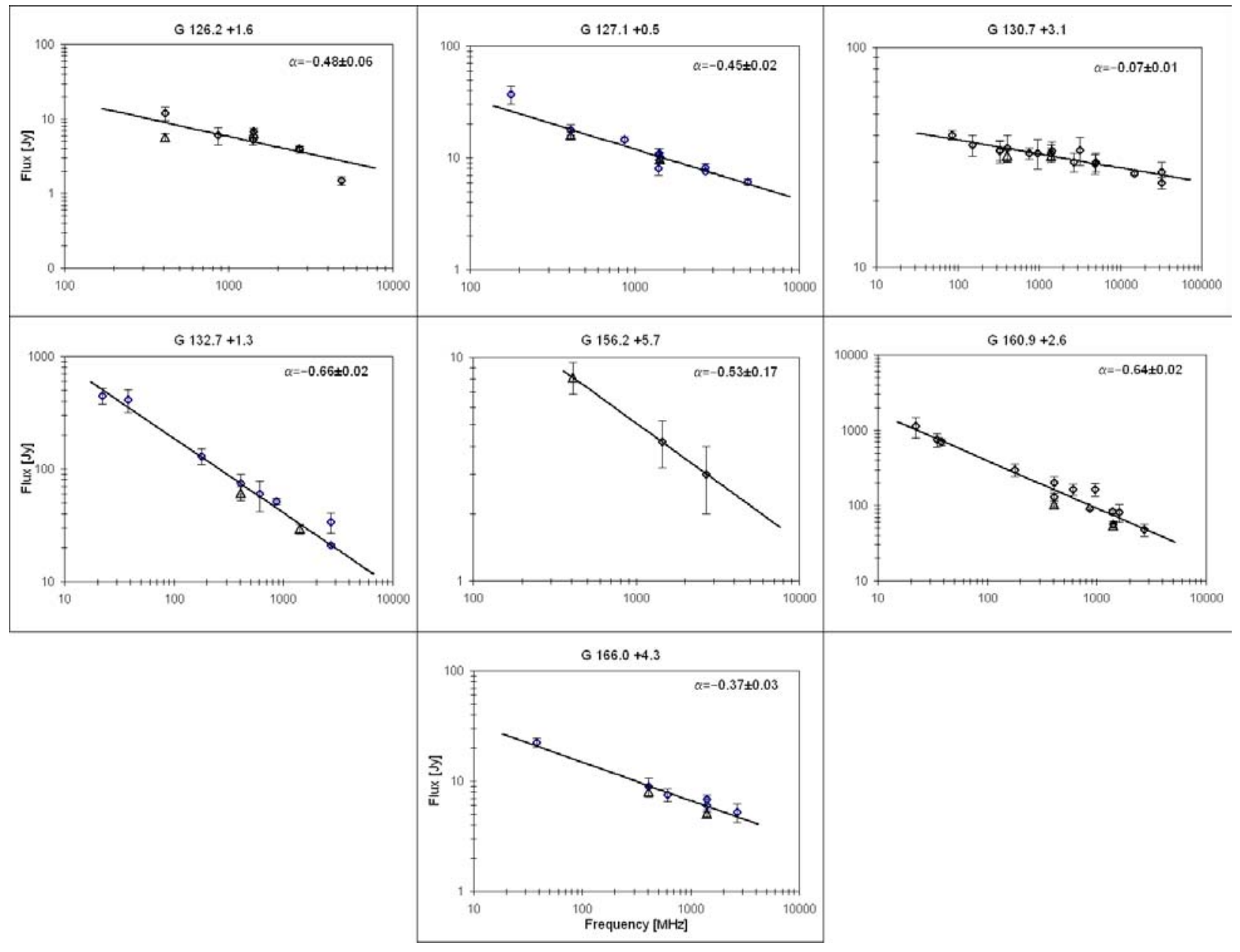

Fig. 10. The radio continuum spectra of the SNRs G126.2+1.6, G127.1+0.5 (R 5), G130.7+3.1 (3C 58, SN 1181) G132.7+1.3 (HB 3), G156.2+5.7, G160.9+2.6 (HB 9), and G166.0+4.3 (VRO 42.05.01). The CGPS flux densities are represented by triangles. 